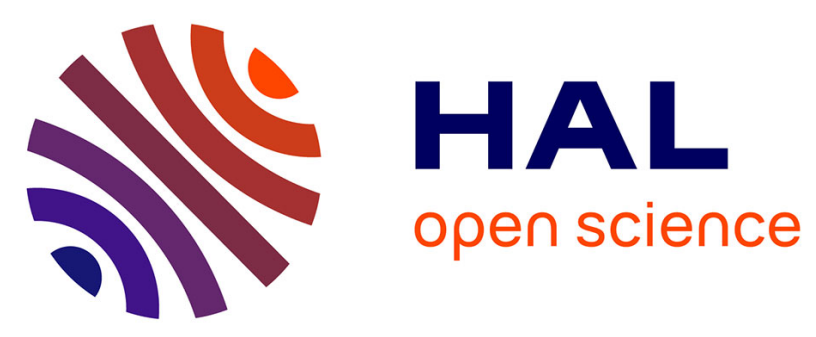

\title{
Model Study of Penetration of Cl- Ions from Solution into Organic Self-Assembled-Monolayer on Metal Substrate: Trends and Modeling Aspects
}

Anton; Kokalj, Dominique Costa

\section{- To cite this version:}

Anton; Kokalj, Dominique Costa. Model Study of Penetration of $\mathrm{Cl}-$ Ions from Solution into Organic Self-Assembled-Monolayer on Metal Substrate: Trends and Modeling Aspects. Journal of The Electrochemical Society, 2021, 168, 10.1149/1945-7111/ac0a24 . hal-03453307

\section{HAL Id: hal-03453307 https://hal.science/hal-03453307}

Submitted on 28 Nov 2021

HAL is a multi-disciplinary open access archive for the deposit and dissemination of scientific research documents, whether they are published or not. The documents may come from teaching and research institutions in France or abroad, or from public or private research centers.
L'archive ouverte pluridisciplinaire HAL, est destinée au dépôt et à la diffusion de documents scientifiques de niveau recherche, publiés ou non, émanant des établissements d'enseignement et de recherche français ou étrangers, des laboratoires publics ou privés. 


\section{Model Study of Penetration of Cl- Ions from Solution into Organic Self- Assembled-Monolayer on Metal Substrate: Trends and Modeling Aspects}

To cite this article before publication: Anton Kokalj et al $2021 \mathrm{~J}$. Electrochem. Soc. in press https://doi.org/10.1149/1945-7111/ac0a24

\section{Manuscript version: Accepted Manuscript}

Accepted Manuscript is "the version of the article accepted for publication including all changes made as a result of the peer review process, and which may also include the addition to the article by IOP Publishing of a header, an article ID, a cover sheet and/or an 'Accepted

Manuscript' watermark, but excluding any other editing, typesetting or other changes made by IOP Publishing and/or its licensors"

This Accepted Manuscript is $\odot 2021$ The Author(s). Published by IOP Publishing Ltd..

As the Version of Record of this article is going to be/has been published on a gold open access basis under a CC 4.0 licence, this Accepted Manuscript is available for reuse under the applicable CC licence immediately.

Everyone is permitted to use all or part of the original content in this article, provided that they adhere to all the terms of the applicable licence referred to in the article - either https://creativecommons.org/licenses/by/4.0/ or https://creativecommons.org/licenses/by-nc-nd/4.0/

Although reasonable endeavours have been taken to obtain all necessary permissions from third parties to include their copyrighted content within this article, their full citation and copyright line may not be present in this Accepted Manuscript version. Before using any content from this article, please refer to the Version of Record on IOPscience once published for full citation and copyright details, as permissions may be required. All third party content is fully copyright protected and is not published on a gold open access basis under a CC licence, unless that is specifically stated in the figure caption in the Version of Record.

View the article online for updates and enhancements. 


\section{Model Study of Penetration of $\mathrm{Cl}-$ Ions from Solution into Organic Self-Assembled-Monolayer on Metal Substrate: Trends and Modeling Aspects}

\begin{tabular}{|c|c|}
\hline Journal: & Journal of The Electrochemical Society \\
\hline Manuscript ID & JES-104309.R2 \\
\hline Manuscript Type: & Research Paper \\
\hline $\begin{array}{r}\text { Date Submitted by the } \\
\text { Author: }\end{array}$ & 04-Jun-2021 \\
\hline Complete List of Authors: & $\begin{array}{l}\text { Kokalj, Anton; Institut Jožef Stefan, } \\
\text { Costa, Dominique; Institut de Recherche de Chimie Paris, }\end{array}$ \\
\hline Keywords: & Corrosion Inhibitors, Corrosion modeling, Theory and Modelling \\
\hline
\end{tabular}




\title{
Model study of penetration of $\mathrm{Cl}^{-}$ions from solution into organic self-assembled-monolayer on metal substrate: trends and modeling aspects
}

\author{
Anton Kokalj ${ }^{\mathrm{a}, *}$, Dominique Costa ${ }^{\mathrm{b}}$ \\ ${ }^{a}$ Department of Physical and Organic Chemistry, Jožef Stefan Institute, Jamova 39, SI-1000 Ljubljana, Slovenia \\ ${ }^{b}$ PSL Research University, CNRS - Chimie ParisTech, Institut de Recherche de Chimie Paris (IRCP), Physical \\ Chemistry of Surfaces Group, 75005 Paris, France
}

\section{Abstract}

Organic molecules that form self-assembled-monolayers on metal substrates may provide efficient corrosion protection. Herein we study how such self-assembled-monolayers hinder the penetration of $\mathrm{Cl}^{-}$ions from aqueous solution toward the metal substrate. We first elucidate some aspects that are relevant for modeling charged ions near surfaces with slab models that utilize periodic-boundary-conditions, in particular: (i) solvation effects, (ii) inter-ion electrostatics, (iii) depolarization effects, and (iv) effects of periodic-boundary-conditions along lateral directions and, for multi-slab models, also along the surface normal direction. The last two effects are artifacts hence they can be avoided or at least minimized by proper modeling. We further present a simple scheme that describes the activation barrier for $\mathrm{Cl}^{-}$penetration into self-assembledmonolayer as a function of the electrode potential and show that the activation barrier decreases as the electrode potential increases, as would be intuitively expected, however, for thick selfassembled-monolayers the barrier remains sizable even at rather positive potentials, which may be one of the reasons why dense and sufficiently thick self-assembled-monolayers can efficiently inhibit corrosion. By utilizing a simple model where metal substrate, organic layer, and aqueous solvent are described implicitly by dielectric continuum slabs, we analyze two important effects by which self-assembled-monolayers hinder the penetration of $\mathrm{Cl}^{-}$ions toward the metal substrate. The first effect is due to inferior solvation of ions in organic layer compared to that in aqueous solvent and the estimated difference is larger than $1 \mathrm{eV}$. This effect is independent of the thickness of the organic layer, provided that the layer is sufficiently thick ( $\gtrsim 10 \AA$ ). The second effect is due to electric field at the electrochemical interface and the extent by which it affects the penetration of $\mathrm{Cl}^{-}$depends on the electrode potential and on the thickness of the organic layer. Other effects, such as local deformation of organic layer during $\mathrm{Cl}^{-}$penetration, cannot be described by current simple models and will be considered in our next publication. Finally, calculations indicate that due to stronger solvation of $\mathrm{Na}^{+}$counter-ions their penetration into organic layer is inferior to that of $\mathrm{Cl}^{-}$. Energetically the most favorable way for $\mathrm{Na}^{+}$to penetrate is in the form of $\mathrm{Na}^{+} / \mathrm{Cl}^{-}$ion-pairs, but it is inferior to penetration of $\mathrm{Cl}^{-}$alone.

${ }^{*}$ Tel: +386-1-477-35-23; Fax: +386-1-251-93-85; Email: tone.kokalj@ijs.si; URL: http://www.ijs.si/ijsw/K3en/Kokalj; ORCID ID: 0000-0001-7237-0041.

Preprint submitted to Journal of The Electrochemical Society 


\section{Introduction}

Localized corrosion by chlorides is a major issue ${ }^{1}$ and chromates have been traditionally used to mitigate the problem. However, due to their carcinogenicity they have been banned and a replacement is needed. Organic corrosion inhibitors represent an attractive option and several families of compounds have been proposed to efficiently inhibit corrosion of $\mathrm{Al}$ and its alloys. In our previous publications we have shown that gallic acid, ${ }^{2}$ silanoles, ${ }^{3,4}$ phosphonates, ${ }^{5}$ and $n$-alkyl carboxylic acids ${ }^{6-8}$ are able to adsorb on oxidized Al surfaces. Among them, $n$-alkyl carboxylic acids (CAs) were shown to be able to efficiently inhibit corrosion of Al, provided that their alkyl chains are long enough. ${ }^{6-8}$ With aid of density-functional-theory (DFT) modeling it was shown that the stability of the CA self-assembled-monolayer (SAM) depends on the adhesion of the carboxylic headgroup to the surface and on the lateral cohesion between alkyl chains, ${ }^{6,7}$ the latter being proportional to the number of $\mathrm{C}$ atoms in the alkyl chain. Consequently, the adsorption is stabilized by about $1 \mathrm{eV} /$ molecule at full monolayer coverage as passing from CA-2 to CA-18, ${ }^{7}$ where the suffix number indicates the number of $\mathrm{C}$ atoms in the CA molecule. These studies provided a sound explanation of why CAs with longer alkyl chains are better corrosion inhibitors than short chain CAs. ${ }^{6,7}$ In particular, longer alkyl chains result in a more stable molecular film on the surface and its greater stability provides a larger thermodynamic force for displacing carbonaceous contamination from the surface. Furthermore, due to molecular tilting the longer alkyl chains cover the surface more effectively than short chains, in particular, for coverages that are nominally too small for the carboxylic head-groups to fully cover the surface.

A step forward in understanding the corrosion inhibition action of CAs would be to study how and to what extent the organic SAMs are able to hinder the penetration of $\mathrm{Cl}^{-}$ions toward the metal substrate. While the fundamental mechanisms responsible for pitting corrosion that involve localized passive film breakdown remain debated, ${ }^{9-11}$ the mechanism of chloride adsorption and penetration into the oxide film on $\mathrm{Al}$ is supported by experimental evidence. ${ }^{12}$ Moreover, even mechanisms that do not attribute to adsorption and penetration of chlorides the seminal cause of passivation breakdown, such as the point defect model (PDM) or the film breakdown mechanism (FBM), include, at some point, either the penetration of $\mathrm{Cl}^{-}$into the oxide film (PDM) - an aspect recently studied computationally using $\mathrm{DFT}^{13}$ —or the adsorption of chlorides at places where the passive film is weakened (FBM). Here also, this aspect has been recently investigated using DFT, showing a clear trend between a chloride susceptibility index and the repassivation potential. ${ }^{14}$ Thus, whatever the involved mechanism, the question of the availability of chlorides at the oxide surface is relevant and, consequently, the role of inhibitors to impede $\mathrm{Cl}^{-}$penetration as well. The purpose of the present paper is not to prove this premise, but rather to explore its consequences, that is, by what mechanism SAMs inhibit the penetration of chlorides toward the metal substrate. If such a hindrance is substantial, then SAM layers could efficiently reduce the chloride-induced pitting.

However, due to diverging long-range Coulomb electrostatic interactions, the modeling of charged "objects" is difficult when periodic-boundary-conditions (PBC) are used and should be exercised with care. Coulomb electrostatic interactions scales as $R^{-1}$, where $R$ is the distance between ions. For a 3D PBC system, which is infinite, summing (integrating) these interactions over all the space gives a diverging $R^{2}$, where $R \rightarrow \infty$. Only when the system is charge neutral, the interactions become conditionally convergent, ${ }^{15}$ because for ionic charge neutral system the electrostatic interactions can be approximated by dipole-dipole interactions and dipole-dipole interactions scale as $R^{-3}$. Even if the surface is modeled as a 2D PBC system, the Coulomb electrostatic interactions are still divergent, because their summation (integration) scales as $R$,

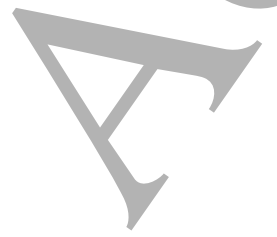


and the system should be made charge-neutral to prevent divergences. For example, in the current work we want to study the penetration of $\mathrm{Cl}^{-}$anion from the aqueous phase into the organic SAM that covers the metal substrate. A way to achieve the charge neutrality of the supercell is to add also a counter-ion, for example $\mathrm{Na}^{+}$. But this typically leads to strong electric fields, because the supercells used in DFT calculations are relatively small, and consequently, the concentration of ions is high.
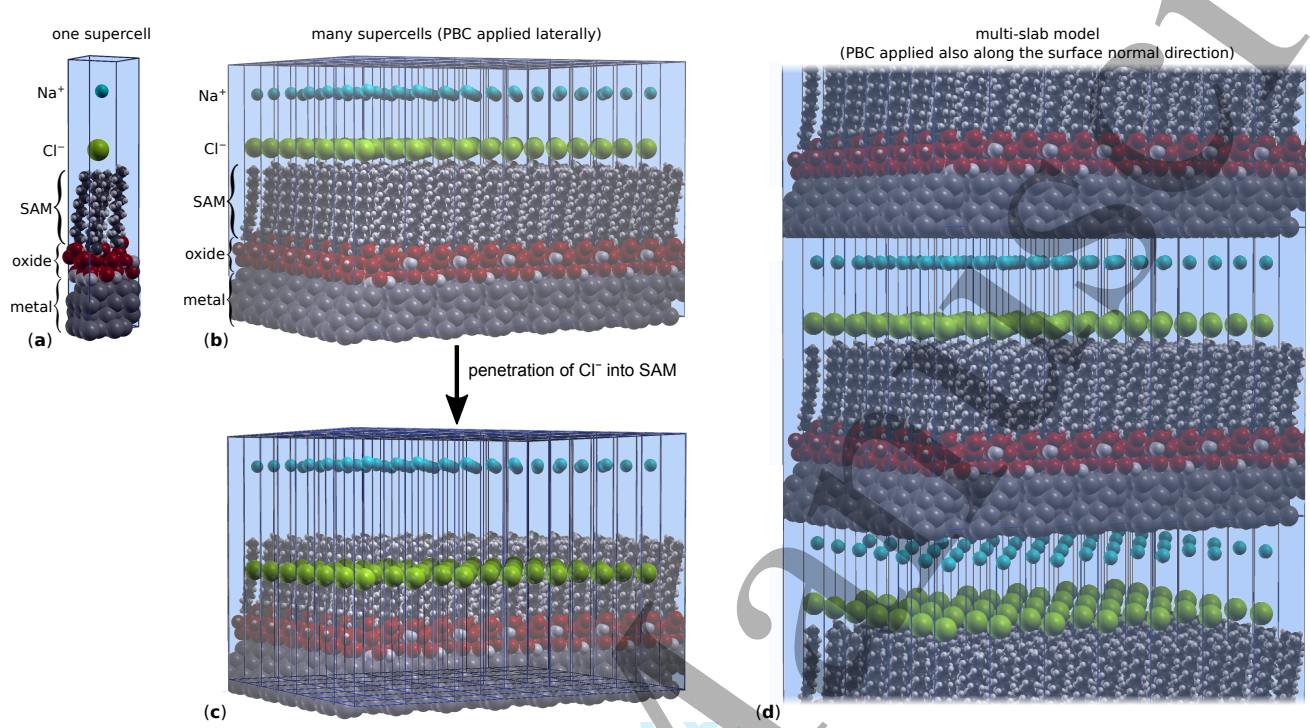

Figure 1. A typical way to model surfaces by DFT methods is to utilize periodic-boundary-conditions (PBC) by means of periodic (multi) slab models. In a slab model, the system is described with a supercell that is repeated periodically in lateral directions, whereas in multi-slab models the PBC are applied also in the surface normal direction. The figure shows how a penetration of $\mathrm{Cl}^{-}$into $\mathrm{SAM} /$ substrate system would be studied by DFT methods; to ensure the charge neutrality a Na${ }^{+}$counter-ion is also added to the supercell. (a) One supercell and (b) many supercells with $\mathrm{Cl}^{-}$ions just above SAM and with (c) $\mathrm{Cl}^{-}$ions entering into SAM. (d) With plane-wave DFT codes surfaces are typically modeled with multi-slab models where periodic-boundary-conditions are applied also in the surface normal direction.

To illuminate the problem, let us consider how surfaces are usually modeled by DFT methods. They are modeled by periodic (multi) slab models. In a slab model, the system is described with a supercell (Fig. 1a) that is repeated periodically in lateral directions (Fig. 1b,c), whereas in a multi-slab model, which is typically utilized when using the plane-wave DFT codes, the PBC are applied also in the surface normal direction (Fig. 1d). Note that the lateral directions are almost always chosen to be along the $x y$ plane and therefore the $z$ direction is the surface normal direction (this choice of directions is also used herein). To study the penetration of $\mathrm{Cl}^{-}$ anion into the organic SAM that covers the metal substrate, the supercell can be constructed as shown in Fig. 1a. The shown supercell consists of about 240 atoms and is composed of an Al metal slab, located at the bottom of the supercell, that supports a hydroxylated native ultra-thin Al-oxide film. An organic SAM, composed of CA-12 molecules, is adsorbed over the oxide-layer. Above the SAM layer a $\mathrm{Cl}^{-}$anion is solvated in an implicit aqueous solvent and a $\mathrm{Na}^{+}$counter-ion is also added to the solvent phase as to ensure the charge neutrality of the supercell. With such a setup, however, one does not study a single $\mathrm{Cl}^{-}$anion, but rather an infinite 2D layer of them as can be seen from Fig. 1b where the supercell is multiplied in the lateral directions. One $\mathrm{Cl}^{-}$anion and one $\mathrm{Na}^{+}$counter-ion per supercell therefore form two layers

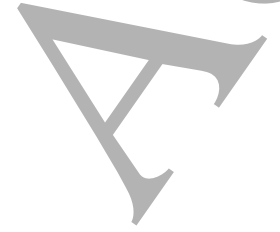




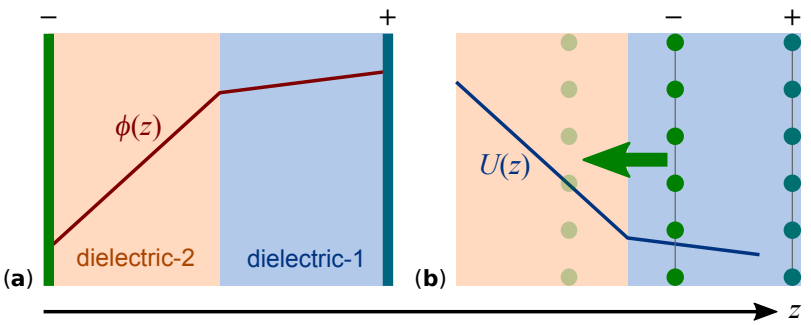

Figure 2. The double-layer of $\mathrm{Cl}^{-}$and $\mathrm{Na}^{+}$ions shown in Fig. 1b,c can be approximately described by a parallel plate capacitor. (a) Electric potential $\phi$ in the parallel plate capacitor with two dielectrics in between the plates and (b) potential energy $U$, as given by the parallel plate capacitor model, as the $\mathrm{Cl}^{-}$layer moves away from the $\mathrm{Na}^{+}$layer. The electric potential and potential energy within a given dielectric are linear with the slope proportional to $\sigma / \varepsilon$, where $\sigma$ is the magnitude of the surface charge density and $\varepsilon$ is the relative static permittivity of a dielectric.

of charges that are reminiscent of a parallel plate capacitor with a strong electric field between the two layers of charges. As the $\mathrm{Cl}^{-}$ion is moved in the simulation from aqueous solvent into organic SAM, it is the whole infinite $\mathrm{Cl}^{-}$layer that moves inward, as illustrated in Fig. 1b,c. But such a movement of the whole $\mathrm{Cl}^{-}$layer is an artifact of the modeling and due to a strong electric field between the two layers this modeling artifact can substantially affect the energy barrier for $\mathrm{Cl}^{-}$penetration into SAM (electric potential in the parallel plate capacitor and the corresponding change of potential energy as the two plates are moved away from each other are schematically shown in Fig. 2). While strong electric fields indeed develop due to the formation of a doublelayer at electrochemical interfaces, the double-layer created by the aforementioned layers of $\mathrm{Cl}^{-}$ and $\mathrm{Na}^{+}$ions seems nevertheless artificial. In reality, dealing with localized corrosion, it is more likely that $\mathrm{Cl}^{-}$ions penetrate a SAM individually, that is, a given ion penetrates at a given time here and another ion at another time there.

Furthermore, with plane-wave DFT codes the surfaces are usually modeled with the multislab model, where the PBC conditions are applied also in the surface normal direction. This implies that slabs are stacked one on top of the other in the surface normal direction and in between a "thick" vacuum or implicit solvent layer is inserted (Fig. 1d) as to prevent chemical interactions between adjacent slabs. In contrast to short-range chemical interactions, in PBC calculations the long-range Coulombic interactions between charged "objects" cannot be made small by increasing the vacuum (or solvent) thickness. For example, if a $\mathrm{Cl}^{-} / \mathrm{Na}^{+}$double-layer is studied by a 3D multi-slab model, then the potential energy changes parabolically and not linearly with the interlayer distance and if this effect is not corrected by some means then this results in yet another artifact.

The purpose of this paper is to disentangle the aforementioned artifacts from the real effects by means of simple theory as well as model calculations and once the artifacts are dealt with to scrutinize the real effects by which organic SAM can hinder the penetration of $\mathrm{Cl}^{-}$ions to the metal substrate. To this end, a simple scheme is developed that describes the activation barrier for $\mathrm{Cl}^{-}$penetration into SAM as a function of the electrode potential.

\section{Technical details}

DFT calculations and implicit description of dielectrics.-DFT calculations were performed by the PWscr code from the QuANTum ESPRESSO (QE) distribution. ${ }^{16}$ Kohn-Sham orbitals were 
expanded in a plane-wave basis set with a kinetic energy cutoff of 30 Ry (240 Ry for the chargedensity cutoff) and core electrons were described implicitly by the projector augmented wave (PAW) method. ${ }^{17,18}$

Metal substrates and organic SAMs were modeled implicitly as continuum dielectric slabs. For metal a huge relative static permittivity of a few thousands was used. Note that such dielectric models are representative of dielectric liquids, because ions can easily penetrate them without any elastic penalty or Pauli repulsion that would be present in solids. It has to be kept in mind therefore that the results presented herein do not capture these effects. Hence an implicit metal is at best representative of a liquid metal, such as mercury at room temperature. The situation is a bit alleviated for a SAM, because it can be seen as a sort of smectic liquid. In contrast to dielectric slabs, $\mathrm{Cl}^{-}$and $\mathrm{Na}^{+}$ions were modeled explicitly using the Perdew-Burke-Ernzerhof (PBE) functional ${ }^{19}$ (for consistency with the next publication ${ }^{20}$ where the substrate and SAM will be modeled explicitly, the PBE-D2 functional was actually used, where the suffix stands for the Grimme D2 empirical dispersion correction). ${ }^{21}$ A typical supercell used in the calculations therefore consists of only $\mathrm{Cl}^{-}$and $\mathrm{Na}^{+}$ions, whereas the metal/SAM/water system is described implicitly by continuum dielectric slabs.

Solvation of $\mathrm{Na}^{+}$and $\mathrm{Cl}^{-}$ions in dielectrics was modeled with the Environ plugin ${ }^{22}$ for QE. Mainly the soft-sphere-continuum-solvation (SoftCS) method ${ }^{20}$ was used, because it affords easier SCF convergence than the self-consistent-continuum-solvation (SCCS) method, ${ }^{22}$ although few calculations were also performed with the latter. For comparison, a few ion solvation calculations were also performed withe Gaussian $16^{23}$ using the SMD solvent model $^{24}$ and def2TZVP Gaussian basis set. ${ }^{25}$

In some cases $\mathrm{Na}^{+}$and $\mathrm{Cl}^{-}$ions were represented by Gaussian charges, labeled as $Q^{+}$and $Q^{-}$, respectively, where the spread of the $Q^{-}$was fitted to reproduce the calculated solvation free energy of $\mathrm{Cl}^{-}$(the $Q^{-}$spread of $1.67 \AA$ was used). In such cases, a single He atom was added to the supercell far away from $Q^{+}$and $Q^{-}$. The reasons for adding the He atom is to have some electrons in the system, which enables a QE calculation.

Relative static permittivity of SAM layer was estimated by performing explicit PBE-D2 calculations of polyethylene crystal and hypothetical crystal of CA-12 molecules arranged in layers that mimics SAM. Respective calculations were performed with the PHonon code from the QE distribution using the linear response theory. ${ }^{26}$ The so calculated permittivity values for polyethylene and CA-12 are similar, about 2.3, hence this value was used to model implicit SAMs.

As stated above, SAMs were described implicitly as continuum dielectric slabs with permittivity of 2.3, hence SAMs of CA molecules with different alkyl chain lengths were modeled simply by adjusting the thickness of the dielectric SAM slabs to that of the respective explicit SAM models. ${ }^{7,20}$ SAM-18, SAM-12, SAM-9, SAM-6, SAM-3, and SAM-2 were considered, where the suffix number represents the number of $\mathrm{C}$ atoms in the CA molecule (e.g., SAM-12 consists of CA-12 molecules). The following thicknesses of SAMs were used: SAM-18 (25.6 $)$, SAM-12 (18 ̊), SAM-9 (14.2 ̊), SAM-6 (10.4 $)$, SAM-3 (6.6 ̊), and SAM-2 (5.3 $)$.

Model electrostatic point-charge calculations. - In addition to DFT calculations, much simpler model calculations were also performed, where ions were treated as point-charges (labeled as $q^{-}$ and $q^{+}$). The respective electrostatic equations (see below) involve lattice sums, which were calculated by means by purposely written Fortran codes. Furthermore, some equations were solved numerically (also by purposely written simple codes), whereas fitting and graph plotting were performed with the Gnuplot program. ${ }^{27}$ Postprocessing of figures was done in Inkscape ${ }^{28}$ and molecular graphics were generated with xcrysden. ${ }^{29}$

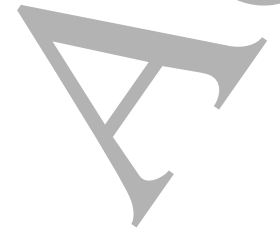




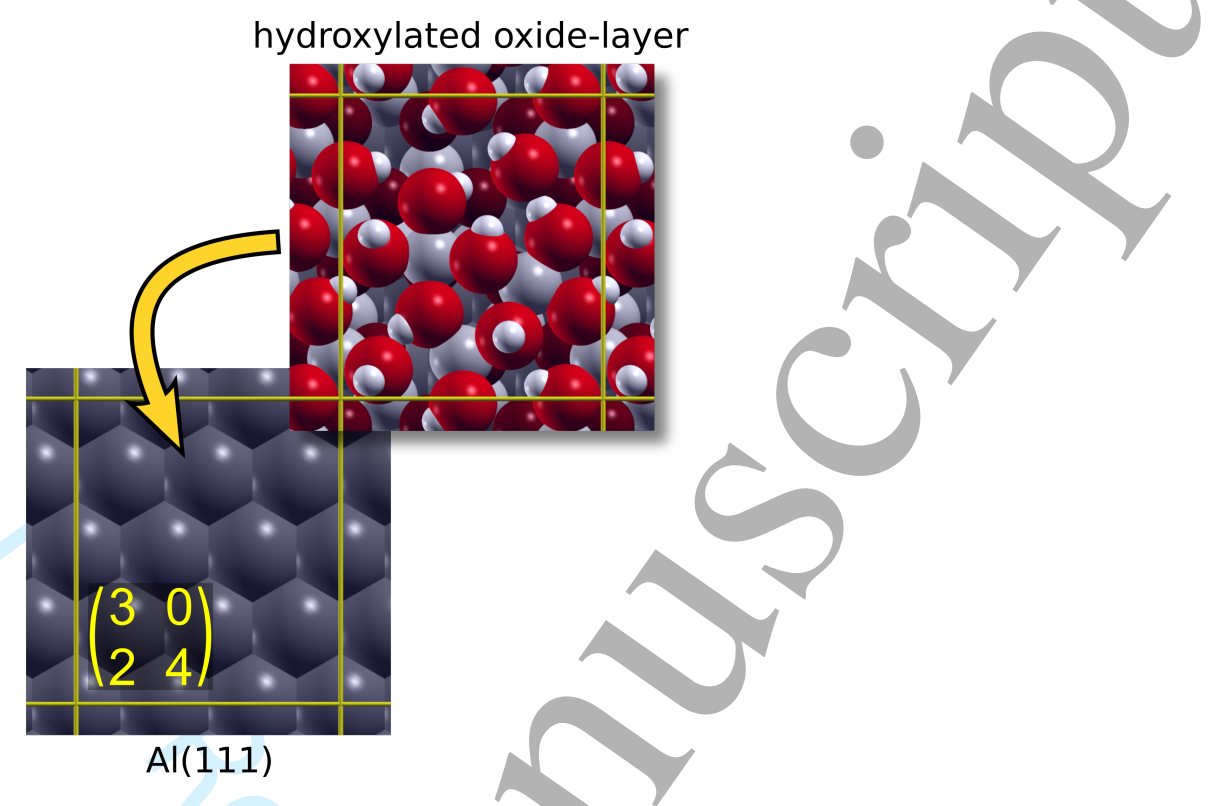

Figure 3. A top-view snapshot of the supercell used in our previous studies ${ }^{6,7}$ to model hydroxylated oxidized Al surface, i.e., the so-called $\mathrm{AlOOH} / \mathrm{Al}_{2} \mathrm{O}_{3} / \mathrm{Al}(111)$ model. The supercell shown in Fig. 1a is also based on this supercell. The supercell designation of $\left(\begin{array}{ll}3 & 0 \\ 2 & 4\end{array}\right)$ corresponds to the underlying $\mathrm{Al}(111)$ support (shown on the bottom-left) that is covered by an ultra-thin hydroxylated oxide film (shown on the top-right). The lateral dimensions of this supercell, labeled as $\mathbf{S}$ or 1S, cf. Eq. [1], are also the basis for the current study.

$2 D$ supercells. - As for the lateral dimensions of the supercells, the $\left(\begin{array}{ll}3 & 0 \\ 2 & 4\end{array}\right)$ supercell of the socalled $\mathrm{AlOOH} / \mathrm{Al}_{2} \mathrm{O}_{3} / \mathrm{Al}(111)$ model, used in our previous studies, ${ }^{6,7}$ will be taken as a reference (Fig. 3); note that this supercell designation refers to the $\mathrm{Al}(111)$ support. This reference supercell is designated by matrix $\mathbf{S}$, that is, $\mathbf{S}=\left(\begin{array}{ll}3 & 0 \\ 2 & 4\end{array}\right)$. Several $(N \times N)$ supercells of this reference supercells will be used herein. Let $\mathbf{N}$ stands for a diagonal matrix, i.e. $\mathbf{N}=\left(\begin{array}{cc}N & 0 \\ 0 & N\end{array}\right)$. We will use the NS supercells, which in crystal-basis can be written as:

$$
\mathbf{N S}=\left(\begin{array}{cc}
N & 0 \\
0 & N
\end{array}\right)\left(\begin{array}{ll}
3 & 0 \\
2 & 4
\end{array}\right)=\left(\begin{array}{cc}
3 N & 0 \\
2 N & 4 N
\end{array}\right)
$$

with $\mathbf{N}$ ranging from $\mathbf{1}$ to $\mathbf{6}$, where, e.g., $\mathbf{6}$ stands for the $\left(\begin{array}{ll}6 & 0 \\ 0 & 6\end{array}\right)$ matrix. The Cartesian representation of the NS supercell can be written as:

$$
\mathbf{N S}=\left(\begin{array}{cc}
3 N & 0 \\
2 N & 4 N
\end{array}\right)\left(\begin{array}{l}
\boldsymbol{a} \\
\boldsymbol{b}
\end{array}\right)
$$

where $\boldsymbol{a}$ and $\boldsymbol{b}$ are the $\mathrm{Al}(111)-(1 \times 1)$ unit-cell vectors, i.e.:

$$
\boldsymbol{a}=\frac{a_{\mathrm{fcc}}}{\sqrt{2}}(1,0) \quad \text { and } \quad \boldsymbol{b}=\frac{a_{\mathrm{fcc}}}{\sqrt{2}}\left(-\frac{1}{2}, \frac{\sqrt{3}}{2}\right)
$$

and $a_{\mathrm{fcc}}$ is the bulk lattice parameter of $\mathrm{Al}$ bulk. By using the PBE-D2 calculated lattice parameter of Al bulk of $4.06 \AA,{ }^{20}$ the NS supercell can be written in Cartesian $\AA$ units as:

$$
\mathbf{N S}=\left(\begin{array}{cc}
N & 0 \\
0 & N
\end{array}\right)\left(\begin{array}{ccc}
8.574 \AA & 0 \\
0 & 9.901 \AA
\end{array}\right) .
$$


The lateral dimensions of the orthogonal $\mathbf{S}$ supercell of $\mathrm{Al}(111)$ are therefore $8.574 \times 9.901 \AA^{2}$, whereas the largest considered $\mathbf{6 S}$ supercell is 36-times larger and has the lateral dimensions of $51.45 \times 59.40 \AA^{2}$.

\section{Theory}

The Hartree atomic units will be used for equations throughout the manuscript, because we find them convenient, although the results will be mainly given in $\AA, \mathrm{eV}$, and $\mathrm{V}$ units. In Hartree atomic units $e=1$ and $4 \pi \varepsilon_{0}=1$, where $e$ is the elementary charge and $\varepsilon_{0}$ is the permittivity of vacuum, hence the interaction energy between two point-charges $\left(q_{1}\right.$ and $\left.q_{2}\right)$ in a dielectric with a relative static permittivity of $\varepsilon$ can be written as:

$$
U=\frac{q_{1} q_{2}}{\varepsilon\left|r_{1}-r_{2}\right|}=\frac{q_{1} q_{2}}{\varepsilon r_{12}}
$$

where $r_{12}$ is the distance between the two charges in Bohr units. This equation implies that the interaction energy between two elementary unit-charges in vacuum at a distance of 1 Bohr is 1 Hartree $=27.21 \mathrm{eV}$. For $N$ point-charges, the electric potential at the position of the pointcharge $i$ due to other charges and the Coulomb interaction energy between $N$ charges can be written as (in Hartree atomic units):

$$
\phi_{i}=\sum_{j \neq i}^{N} \frac{q_{j}}{\varepsilon\left|r_{i}-r_{j}\right|} \quad \text { and } \quad U=\frac{1}{2} \sum_{i=1}^{N} q_{i} \phi_{i} .
$$

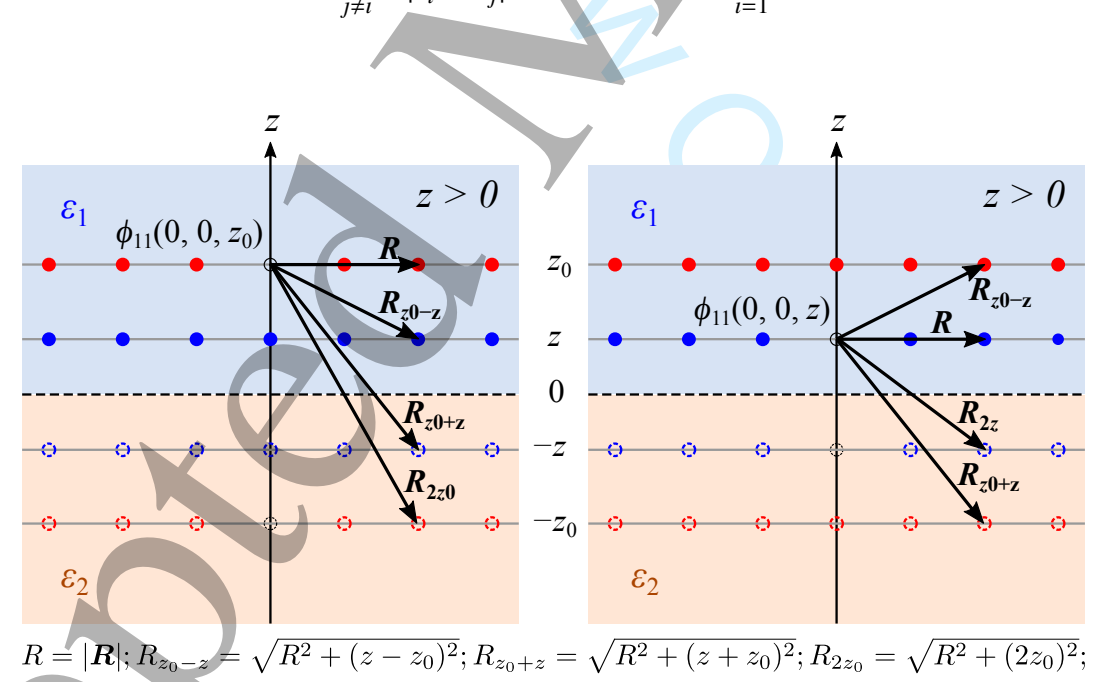

Figure 4. Parallel infinite layers of $q^{+}$and $q^{-}$charges. The layer of positive charges (red solid points) is located at $z_{0}$ in dielectric-1, whereas the layer of negative charges (blue solid points) is beneath it at $z$ and is also located in dielectric-1. Image charges are shown by open dashed circles and arrows indicate various components to the electric potential. Left picture shows the exemplar components contributing to the electric potential at the position of the reference $q^{+}$charge, $\phi_{11}\left(0,0, z_{0}\right)$, and right picture shows analogous for the reference $q^{-}$charge, $\phi_{11}(0,0, z)$. 


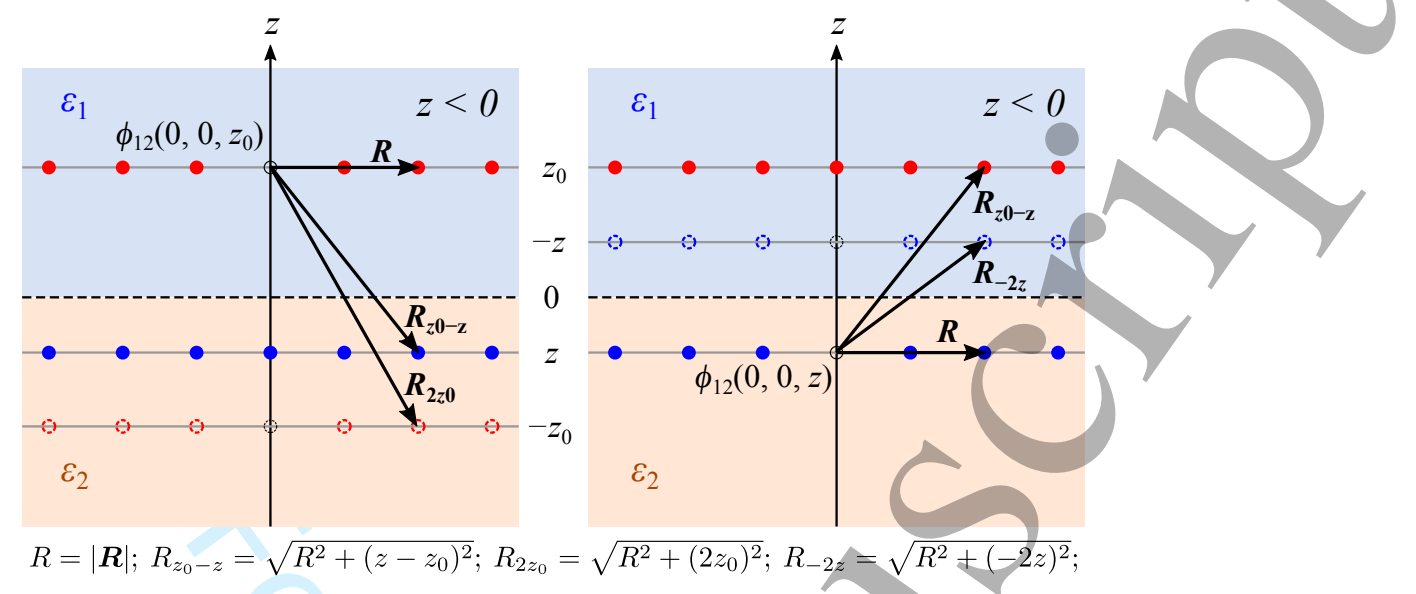

Figure 5. Similarly as in Fig. 4, but for the layer of negative charges (blue solid points) located in dielectric-2 at $z$ (notice that in this case $z<0$ ).

Electrostatic interactions between ions: two infinite parallel layers of point-charges near the interface between two dielectrics. - Let us consider a model system composed of two semiinfinite dielectrics, characterized by $\varepsilon_{1}$ and $\varepsilon_{2}$, and two infinite layers of point-charges, the first consisting of positive charges $\left(q^{+}\right)$and the second consisting of negative charges $\left(q^{-}\right)$. The interface between the two dielectrics lie on the $x y$ plane at $z=0$ and the layer of $q^{+}$is located at $z_{0}$ in dielectric-1, whereas the layer of $q^{-}$is located beneath it at $z$. Such system of pointcharges near the interface between two dielectrics can be déscribed by the method of images (see Appendix). When both layers are in dielectric-1 (Fig. 4), the electric potential at the position of the reference $q^{+}$charge at $\left(0,0, z_{0}\right)$ is:

$$
\phi_{11}\left(0,0, z_{0}\right)=\frac{q^{+}}{\varepsilon_{1}} \sum_{\boldsymbol{R} \neq 0}\left(\frac{1}{R}+\frac{\varepsilon_{1}-\varepsilon_{2}}{\varepsilon_{1}+\varepsilon_{2}} \frac{1}{R_{2 z_{0}}}\right)+\frac{q^{-}}{\varepsilon_{1}} \sum_{\boldsymbol{R}}\left(\frac{1}{R_{z_{0}-z}}+\frac{\varepsilon_{1}-\varepsilon_{2}}{\varepsilon_{1}+\varepsilon_{2}} \frac{1}{R_{z_{0}+z}}\right),
$$

whereas the electric potential at the position of the reference $q^{-}$charge at $(0,0, z)$ is:

$$
\phi_{11}(0,0, z)=\frac{q^{-}}{\varepsilon_{1}} \sum_{\boldsymbol{R} \neq 0}\left(\frac{1}{R}+\frac{\varepsilon_{1}-\varepsilon_{2}}{\varepsilon_{1}+\varepsilon_{2}} \frac{1}{R_{2 z}}\right)+\frac{q^{+}}{\varepsilon_{1}} \sum_{\boldsymbol{R}}\left(\frac{1}{R_{z_{0}-z}}+\frac{\varepsilon_{1}-\varepsilon_{2}}{\varepsilon_{1}+\varepsilon_{2}} \frac{1}{R_{z_{0}+z}}\right),
$$

where the potential is labeled by the 11 subscript to indicate that both layers are in dielectric-1. The lattice sums run over all lattice vectors $\boldsymbol{R}$, except the left sums in Eqs. [7] and [8] exclude the self-interactions (i.e., the $\boldsymbol{R}=\mathbf{0}$ term is omitted). The label $R_{z_{0}+z}$ stands for:

$$
R_{z_{0}+z}=\sqrt{R^{2}+\left(z_{0}+z\right)^{2}}
$$

and the meaning of $R_{2 z_{0}}$ and $R_{2 z}$ is analogous. The interaction energy per ion-pair is therefore:

$$
U_{11}(z)=\frac{1}{2}\left(q^{+} \phi_{11}\left(0,0, z_{0}\right)+q^{-} \phi_{11}(0,0, z)\right)
$$

The interaction energy is written as a function of $z$, because in calculations only the position of $q^{-}$layer at $z$ will be moved, whereas the $q^{+}$layer at $z_{0}$ will be held fixed. 
When the two layers are in different dielectrics, i.e., $q^{+}$layer in dielectric- 1 and $q^{-}$layer in dielectric-2 (Fig. 5), then:

$$
\phi_{12}\left(0,0, z_{0}\right)=\frac{q^{+}}{\varepsilon_{1}} \sum_{\boldsymbol{R} \neq 0}\left(\frac{1}{R}+\frac{\varepsilon_{1}-\varepsilon_{2}}{\varepsilon_{1}+\varepsilon_{2}} \frac{1}{R_{2 z_{0}}}\right)+\frac{2 q^{-}}{\varepsilon_{1}+\varepsilon_{2}} \sum_{\boldsymbol{R}} \frac{1}{R_{z_{0}-z}}
$$

and

$$
\phi_{12}(0,0, z)=\frac{q^{-}}{\varepsilon_{2}} \sum_{\boldsymbol{R} \neq 0}\left(\frac{1}{R}+\frac{\varepsilon_{2}-\varepsilon_{1}}{\varepsilon_{1}+\varepsilon_{2}} \frac{1}{R_{-2 z}}\right)+\frac{2 q^{+}}{\varepsilon_{1}+\varepsilon_{2}} \sum_{\boldsymbol{R}} \frac{1}{R_{z_{0}-z}},
$$

and the interaction energy $U_{12}$ per ion-pair can be calculated analogously to Eq. [10], i.e.,

$$
U_{12}(z)=\frac{1}{2}\left(q^{+} \phi_{12}\left(0,0, z_{0}\right)+q^{-} \phi_{12}(0,0, z)\right)
$$

Here the subscript 12 is used to indicate that the first layer is located in the dielectric- 1 and the second layer in the dielectric-2. There is an important caveat to notice: the subscript, such as $z_{0}-z$ in $R_{z_{0}-z}$, indicates the distance between the two layers and the distance is never negative. This is the reason why the subscript label in Eq. [12] is written as $R_{-2 z}$, because in this case $z<0$ and, therefore, $-2 z>0$.

The interaction energy (per ion-pair) as a function of $z$ for the two parallel infinite layers of $q^{+}$ and $q^{-}$charges can be thus written as:

$$
U(z)= \begin{cases}U_{11}(z) & \text { for } z \geq 0 \\ U_{12}(z) & \text { for } z<0\end{cases}
$$

where $U_{11}(z)$ is given by Eq. [10] and $U_{12}(z)$ by Eq. [13].

The electric potentials $\phi_{11}$ and $\phi_{12}$ involve infinite lattice sums and therefore the dependence of the interaction energies, Eqs. [10] and [13], on the interlayer distance is not trivially obvious. If one is only interested in the main trend then these two equations can be simplified by approximating the lattice sums by integrals (for the corresponding derivation, see Appendix). The resulting approximate equations are:

where

$$
U_{11}^{\text {approx }}=\frac{2 \pi q^{2}}{A \varepsilon_{1}}\left(z_{0}-z\right) \quad \text { and } \quad U_{12}^{\text {approx }}=\frac{2 \pi q^{2}}{A}\left(\frac{z_{0}}{\varepsilon_{1}}-\frac{z}{\varepsilon_{2}}\right),
$$

where $q$ is the magnitude of the point-charge, $q=q^{+}=-q^{-}$, and $A$ is the area of the supercell. The slopes of the interaction energy with respect to $z$ are therefore:

$$
\frac{\partial U_{11}^{\text {approx }}}{\partial z}=-\frac{2 \pi q^{2}}{A \varepsilon_{1}} \propto-\frac{\sigma}{\varepsilon_{1}} \quad \text { and } \quad \frac{\partial U_{12}^{\text {approx }}}{\partial z}=-\frac{2 \pi q^{2}}{A \varepsilon_{2}} \propto-\frac{\sigma}{\varepsilon_{2}}
$$

where $\sigma$ is the magnitude of the surface charge density, $\sigma=q / A$. This implies that approximating Eqs. [10] and [13] by transforming lattice sums into integrals gives the result analogous to that 
of the parallel plate capacitor* (Fig. 2), where the electric potential and potential energy within a given dielectric- $i$ are linear with the slope proportional to $\sigma / \varepsilon_{i}$. With this knowledge, we can generalize the result to the case of multiple dielectric slabs stacked together one on top of the other along the $z$-direction. In each dielectric slab the slope is proportional to the inverse of its permittivity, i.e. $\partial U_{i}^{\text {approx }} / \partial z \propto 1 / \varepsilon_{i}$. Here, it should be noted that the negative slopes of $\partial U_{11}^{\text {approx }} / \partial z$ and $\partial U_{12}^{\text {approx }} / \partial z$ as well as $\partial U_{11} / \partial z$ and $\partial U_{12} / \partial z$ are a consequence of the setup, i.e., the layer of positive charges fixed at $z_{0}$ and the layer of negative charges at $z<z_{0}$ moving away from it in the $-z$ direction. The negative slope is therefore a consequence of the "movement" in the negative direction. The main message is thus that the larger is the interlayer distance, the more positive (endothermic) is the energy.
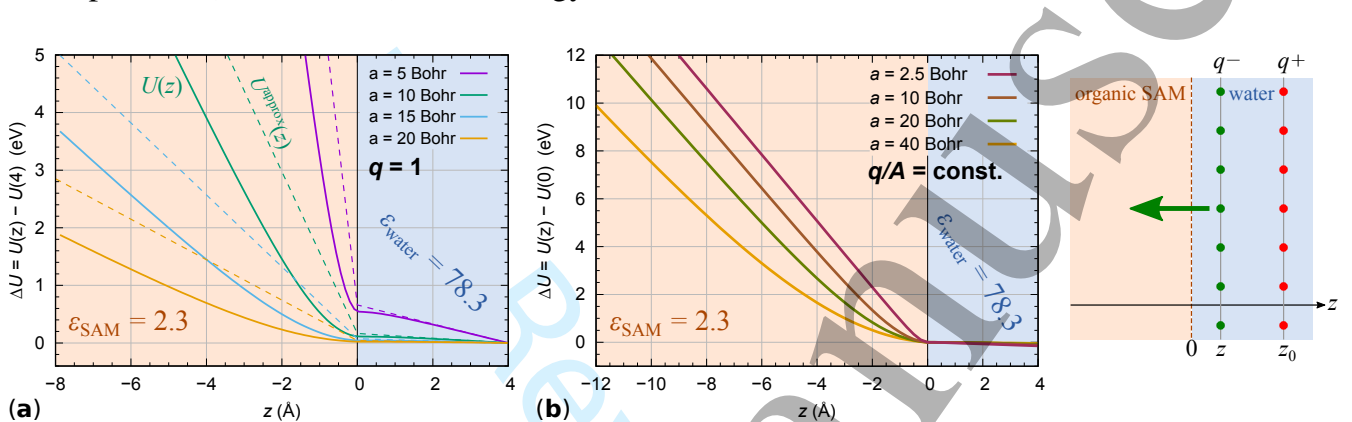

(b)

Figure 6. Analysis of the electrostatic potential energy for two parallel infinite layers of $q^{+}$and $q^{-}$charges near the interface between the organic SAM $\left(\varepsilon_{\mathrm{SAM}}=2.3\right)$ and water $\left(\varepsilon_{\text {water }}=78.3\right)$ dielectrics, $U(z)$ of Eq. [14], for square $2 \mathrm{D}$ unit-cells with various lattice parameters $a$. The $q^{+}$layer is located at $z_{0}=20.6 \AA$ on the far right in water (out of scale), whereas the $q^{-}$layer is located at $z$ (see the schematic on the right). (a) $U(z)$ for various $a$ lattice parameters with charges held constant, $q=q^{+}=-q^{-}=1$. The value of $U(z)$ at $z=4 \AA$ is chosen as the reference zero energy so that all curves start from the same "point". Notice that while $U^{\text {approx }}(z)$ is bilinear (dashed lines) with slope discontinuity at the interface, the explicitly calculated $U(z)$ smoothly changes the slope as going from water into SAM, with the change of slope mainly taking place in the dielectric with the smaller permittivity; this smooth transition can be attributed to interaction with image-charges. (b) $U(z)$ for surface charge density held constant at $\sigma=q / A=0.01 \mathrm{e} / \mathrm{Bohr}^{2}$ and varying the $a$ lattice parameter; here the value of $U(z)$ at the interface at $z=0$ is chosen as the reference zero energy. Note that the smaller is the lattice parameter $a$ the closer is the $U(z)$ to the bilinear behavior of the parallel plate capacitor, $U^{\text {approx }}(z)$ of Eq. [15].

The comparison between $U(z)$ and $U^{\text {approx }}(z)$ is made in Fig. 6a for a bilayer of $q^{+}$and $q^{-}$ charges - several square lattices with various lattice parameters $a$ are considered-near the interface between organic SAM $\left(\varepsilon_{\mathrm{SAM}}=2.3\right)$ and water $\left(\varepsilon_{\text {water }}=78.3\right)$ dielectrics. It can be seen that while $U^{\text {approx }}(z)$ reproduces the $\partial U / \partial z$ slopes far enough from the interface, it fails to reproduce the details near the interface between the two dielectrics. In particular, $U^{\text {approx }}(z)$ is bilinear (dashed lines in Fig. 6a) with slope discontinuity at the interface, whereas the slope of explicitly calculated $U(z)$ smoothly changes from water to SAM with the smooth transition mainly taking place in the dielectric with the smaller permittivity; this smooth transition can be attributed to interaction with image-charges. Fig. 6b compares the $U(z)$ curves for square lattices of constant surface charge density and it can be seen that the smaller is the lattice parameter $a$ the closer is the $U(z)$ curve to the bilinear behavior of the parallel plate capacitor, $U^{\text {approx }}(z)$ of Eq. [15].

There is a factor of 2 difference between the current case and the parallel plate capacitor-i.e., the potential energy of a point-charge inside the parallel plate capacitor is proportional to $4 \pi \sigma q / \varepsilon$-and this difference will be clarified later on.

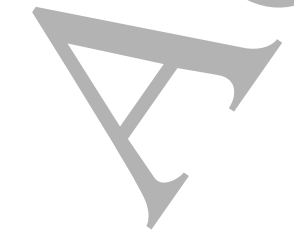


$\mathrm{Na}^{+} / \mathrm{Cl}^{-}$in a supercell
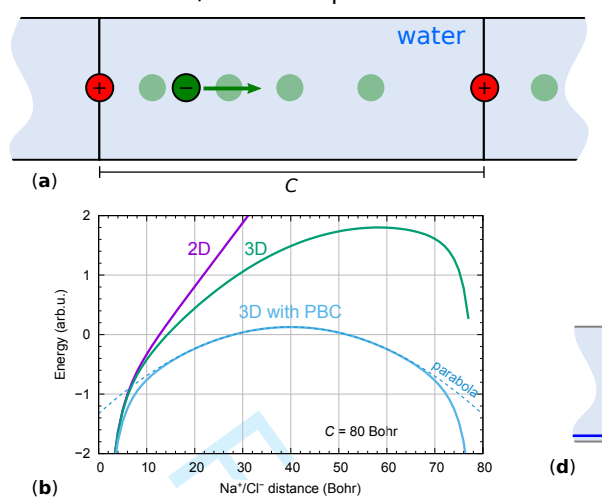

(d)
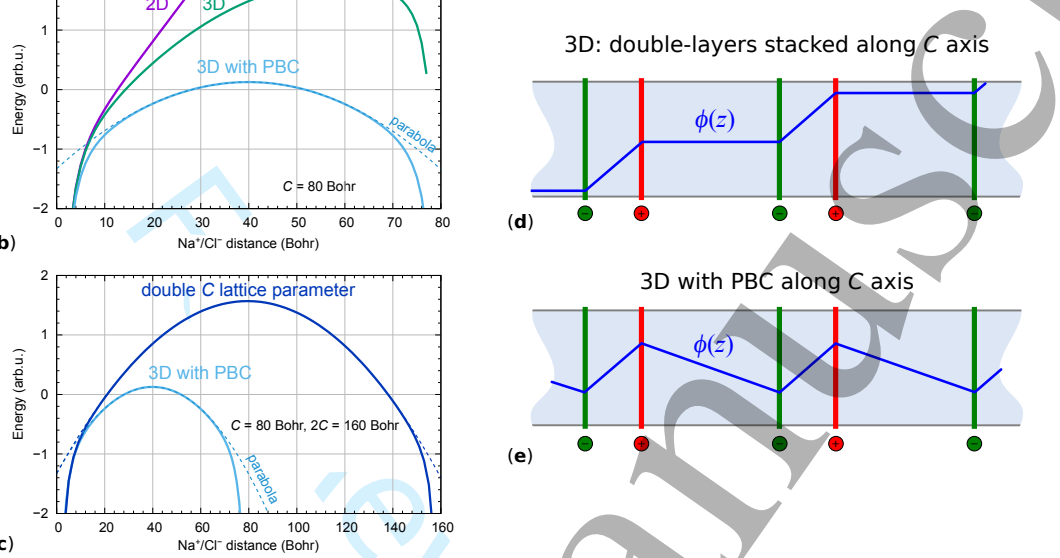

3D with PBC along $C$ axis

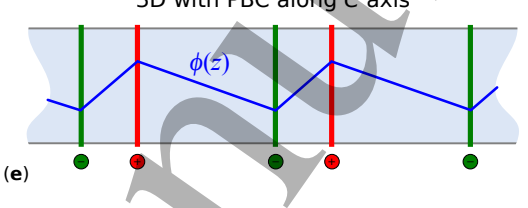

(c)

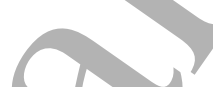

Figure 7. Emulation of a $\mathrm{Na}^{+} / \mathrm{Cl}^{-}$(or $q^{+} / q^{-}$) double-layer with a 3D system. While the $2 \mathrm{D}$ system consists of a single $q^{+} / q^{-}$double-layer, the corresponding 3D system consists of double-layers stacked one on top of the other, such that the stack can be described with the lattice parameter $C$ as shown in (a). (b) Comparison between the interaction energy of the $2 \mathrm{D}$ system versus the 3D system with and without the PBC along the $C$ direction as the layer of $q^{-}$charges moves away from the $q^{+}$layer. Note that for the 2D system the energy increases linearly with the interlayer distance for distances larger than about $10 \mathrm{Bohr}$ (the actual distance where this happens depends on the lateral dimensions of the cell), whereas for the 3D system the energy first increases and then, for large enough distances, starts decreasing because the $q^{-}$layer approaches the adjacent periodic-image layer of $q^{+}$ions. For a 3D system without PBC along the $C$ axis, the interaction energy profile is "tilted" upwards due to a dipole that develops; the corresponding electric potential is shown in (d). Such a profile of electric potential and interaction energy is not compatible with PBC along the $C$ axis, because for the 3D PBC case the electric potential is translationally invariant, as shown in (e), and the interaction energy profile is "symmetric", i.e., $U(z)=U(z+C)$ and $\phi(z)=\phi(z+C)$. Although for 3D PBC the interaction energy profile is parabolic, it is still divergent with respect to the $C$ lattice parameter, as shown in (c) that compares interaction energy profiles for two unit cells with the size of $C$ and $2 C$. It is evident that in the limit of $C \rightarrow \infty$ the interaction energy profile approaches that of the true $2 \mathrm{D}$ system.

Modeling 2D system with 3D setup: $3 D$ PBC artifacts. - As shown above, the case of two infinite layers of opposite charges is easy to understand, i.e., the interaction energy increases linearly with the interlayer distance (for large enough interlayer distances). However, in plane-wave DFT calculations we typically use 3D PBC and what happens when a 2D double-layer system is modeled by $3 \mathrm{D}$ PBC is shown in Fig. 7 for a simple case of a $\mathrm{Na}^{+} / \mathrm{Cl}^{+}$double-layer in water dielectric. Due to 3D PBC, the interaction energy is not linear with the interlayer distance, but it is instead parabolic (for large enough interlayer distances), which is an artifact due to the use of 3D PBC. In particular, for the 3D system the energy first increases as the layer of $q^{-}$charges moves away from the $q^{+}$layer, but then eventually it starts decreasing as the $q^{-}$layer approaches the adjacent periodic-image layer of $q^{+}$ions. For a 3D system without PBC along the $z$-direction, the interaction energy profile is "tilted" upwards (Fig. 7b) due to a dipole that develops. Such an interaction energy profile is not compatible with PBC along the $z$-direction, because for the 

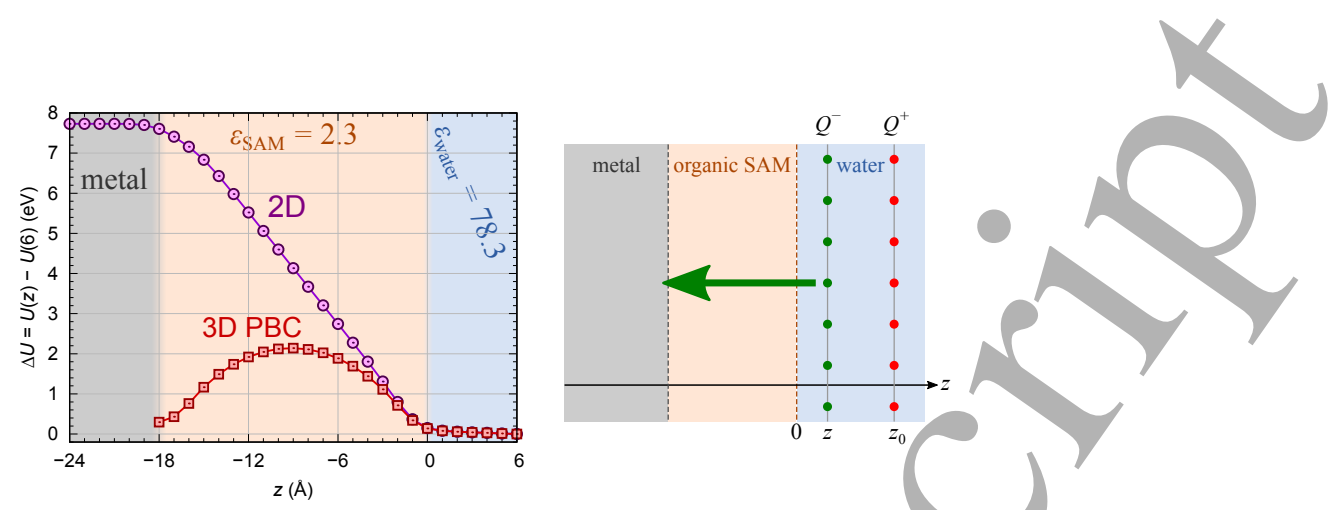

Figure 8. An illustration of $3 \mathrm{D}$ PBC artifacts (red curve) by means of comparison to the corresponding 2D system (purple curve) for the penetration of $Q^{-}$layer from water into the SAM-12/metal substrate. The system consists of a double-layer of $Q^{+} / Q^{-}$Gaussian charges and three implicit dielectric slabs (metal, organic SAM-12, and water, where the metal is modeled as implicit dielectric with a huge permittivity constant of a few thousands). The $Q^{+}$layer is held fixed in water, whereas the $Q^{-}$layer is moved from water, into SAM-12, and finally into metal in the $-z$ direction (the setup is shown schematically on the right). The lateral dimensions of the system are those of the reference $\mathbf{S}$ supercell, defined in Fig. 3 and Eq. [1], which corresponds to a surface charge density of $1.18 \mathrm{e} / \mathrm{nm}^{2}$.

3D PBC case the interaction energy should be translationally invariant, i.e., $U(z)=U(z+C)$, where $C$ is the lattice parameter along the $z$-direction, and as a consequence its profile becomes parabolic (unless the two layers are too close). Although for 3D PBC the interaction energy profile is parabolic, it is still divergent with respect to the $C$ lattice parameter, as shown in Fig. 7c that compares the interaction energy profiles for two unit cells with the size of $C$ and $2 C$ along the $z$-direction. It is evident that in the limit of $C \rightarrow \infty$ the interaction energy profile approaches that of the true $2 \mathrm{D}$ system.

The next relevant question is how the 3D PBC artifacts affect the energy profile of the penetration of " $\mathrm{Cl}^{-}$" layer from water into SAM/metal substrate when the system is modeled without any correction for the 3D PBC artifacts. The answer to this question is provided by Fig. 8, which considers the SAM-12/metal system for the 1S supercell, and the result is astounding: the system simply cannot be modeled with the 3D PBC setup if no correction is made! Note that only for the first several $\AA$ into SAM the 3D PBC interaction energy follows the 2D case, but then the parabolic profile of 3D PBC starts deviating considerably from the linear 2D case and as a consequence the predicted barrier is "only" about $2 \mathrm{eV}$ for the currently considered case, whereas the $2 \mathrm{D}$ calculation gives a barrier of about $8 \mathrm{eV}$.

A simple, though not computationally efficient, method to get rid of 3D PBC artifacts is to use a "symmetric" setup, where the metal $/\left\{\mathrm{SAM}, \mathrm{Cl}^{-}\right\} /\left\{\right.$water, $\left.\mathrm{Na}^{+}\right\}$system is described by a doublesided symmetric $\left\{\mathrm{Na}^{+}\right.$, water $\} /\left\{\mathrm{Cl}^{-}, \mathrm{SAM}\right\} /$ metal $/\left\{\mathrm{SAM}, \mathrm{Cl}^{-}\right\} /\left\{\right.$water, $\left.\mathrm{Na}^{+}\right\}$model. This way the dipole created by the $\mathrm{Na}^{+} / \mathrm{Cl}^{-}$double-layer above the metal slab is canceled by the $\mathrm{Cl}^{-} / \mathrm{Na}^{+}$ double-layer below the metal slab. Other possibility is, for example, to use a dipole correction that inserts a counter double-layer above the layer of $\mathrm{Na}^{+}$ions but below the adjacent periodic image of metal slab, where the dipole of the counter double-layer is self-consistently adjusted to cancel the dipole of the metal/ $\left\{\mathrm{SAM}, \mathrm{Cl}^{-}\right\} /\left\{\right.$water, $\left.\mathrm{Na}^{+}\right\}$system. Herein the "symmetric" setup method was used for the QE calculations as to get rid of the 3D PBC artifacts, whereas the point-charge calculations were performed by true $2 \mathrm{D}$ systems.

Depolarization/deionization effects. - Fig. 8 reveals that for the concentration of ions as given by the reference $\mathbf{S}$ supercell, which corresponds to a surface charge density of $1.18 \mathrm{e} / \mathrm{nm}^{2}$, the

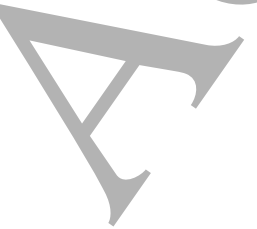



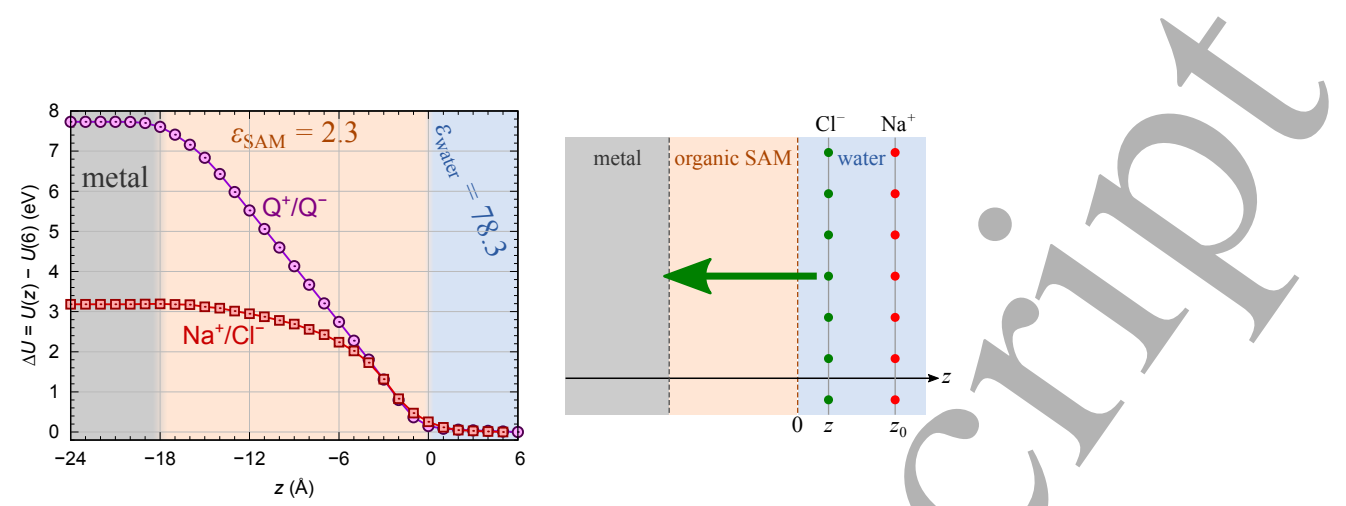

Figure 9. Comparison of the interaction energy profiles for the double-layer of $Q^{+} / Q^{-}$Gaussian charges and doublelayer of $\mathrm{Na}^{+} / \mathrm{Cl}^{-}$ions for the penetration of the negatively-charged layer $\left(Q^{-}\right.$or $\left.\mathrm{Cl}^{-}\right)$from water into the $\mathrm{SAM} / \mathrm{meta}$ substrate, where the water, SAM, and metal slabs are modeled by implicit dielectrics analogously to that of Fig. 8. The positively charged counter-layer is held fixed in water (the setup is shown on the right). The lateral dimensions of the system are those of the reference $\mathbf{S}$ supercell. Notice that the $\mathrm{Na}^{+} / \mathrm{Cl}^{-}$system depolarizes for $z \lesssim-6 \AA$ as to reduce the energy.

interaction energy raises almost up to $8 \mathrm{eV}$ as the $Q^{-}$layer penetrates from water through SAM12 toward the metal. Due to the large surface charge density, the electric field in the SAM is huge and the energy difference of about $8 \mathrm{eV}$ is so large that "depolarization" occurs when the system is modeled by the actual $\mathrm{Cl}^{-} / \mathrm{Na}^{+}$ions, that is, the $\mathrm{Cl}^{-}$and $\mathrm{Na}^{+}$ions partially de-ionize as to lower the energy; this effect is evidenced by Fig. 9. Due to this "depolarization" the barrier for the penetration reduces from about $8 \mathrm{eV}$ to $3 \mathrm{eV}$. The comparison between these two barriers also reveals that to prevent the depolarization the supercell should be at least about 3 times larger (i.e., $8 / 3 \approx 3$ ) than the reference $\mathbf{S}$ supercell, defined in Fig. 3 . This suggests, for example, that the $\mathbf{2} \mathbf{S}$ supercell, Eq. [1], should be already applicable for modeling SAM-12, because it is four-times larger than $\mathbf{S}$ (note that the minimal size of the supercell, which prevents depolarization, depends on the thickness of SAM, i.e., thicker SAMs require larger supercells).

It should be also noted that the large slope of the interaction energy in SAM, shown in Fig. 9, is not only responsible for the depolarization of $\mathrm{Na}^{+} / \mathrm{Cl}^{-}$double-layer, but also masks another important effect, that is, the change in solvation free energy as the $\mathrm{Cl}^{-}$moves from water into SAM. This effect is analyzed below.

Solvation effects. - Another important aspect of the $\mathrm{Cl}^{-}$penetration from water into an organic SAM are solvation effects. According to the simple Born solvation model, the free energy of solvation depends on the ion radius $r_{\text {ion }}$ and the medium permittivity $\varepsilon$ and can be approximated as (in Hartree atomic units):

$$
\Delta G_{\mathrm{solv}}^{\mathrm{Born}}=-\frac{q_{\mathrm{ion}}^{2}}{2 r_{\text {ion }}}\left(1-\frac{1}{\varepsilon}\right),
$$

where $q_{\mathrm{ion}}$ is the charge of the ion. This model allows to approximately estimate the difference in solvation of $\mathrm{Cl}^{-}$in water and organic SAM. The radius of $\mathrm{Cl}^{-}$ion is $1.81 \AA$ (3.42 Bohr), whereas permittivities of water and SAM are 78.3 and 2.3 , respectively. Hence:

$$
\Delta \Delta G_{\text {solv }}^{\text {Born }}=\Delta G_{\text {solv }}^{\text {Born }}(\mathrm{SAM})-\Delta G_{\text {solv }}^{\text {Born }}(\text { water })=-2.2-(-3.9) \mathrm{eV}=1.7 \mathrm{eV} \text { for } \mathrm{Cl}^{-} .
$$

Table I reports experimental and calculated solvation free energies for $\mathrm{Cl}^{-}$and $\mathrm{Na}^{+}$ions in water, whereas the calculated data for solvation in SAM as well as the $\Delta \Delta G_{\text {solv }}$ differences are given in 


\section{Table I}

Solvation free energies (in $\mathrm{eV}$ ) for $\mathrm{Cl}^{-}$and $\mathrm{Na}^{+}$ions in water $\left(\Delta G_{\text {solv }}^{\text {real }}\right.$ is the real solvation free energy). Experimental values and values calculated with various continuum solvation models are given. Data labeled with "g16 SMD" were calculated with Gaussian16 using the SMD implicit solvent model and data labeled with QE were calculated with Environ plugin of QUANTUM ESPRESSO; SCCS refers to self-consistent-continuum-solvation method ${ }^{22}$ and SoftCS to softsphere-continuum-solvation method. ${ }^{30}$ Labels "water", "water-anion", and "water-cation" refer to various parametrizations, in particular, "water-anion" and "water-cation" are Environ parametrizations specifically tuned for anions and cations, respectively. Note that g16 SMD predicts too weak and QE too strong hydration of $\mathrm{Na}^{+}$, whereas for $\mathrm{Cl}^{-}$the calculated values are much closer to the experimental value.

\begin{tabular}{llcc}
\hline Method & $\begin{array}{c}\mathrm{Cl}^{-} \\
(\mathrm{eV})\end{array}$ & $\begin{array}{c}\mathrm{Na}^{+} \\
(\mathrm{eV})\end{array}$ \\
\hline$\Delta G_{\text {solv }}^{\text {real }}$ & expt. $\left(\right.$ Ref. $\left.^{31}\right)$ & -3.08 & -4.28 \\
$\left.\Delta G_{\text {solv }}\right)$ & expt. $\left(\right.$ Ref. $\left.^{31}\right)$ & -3.30 & -4.28 \\
& g16 SMD & -2.92 & -3.13 \\
& QE water SCCS & -2.89 & -5.59 \\
QE water-anion SCCS & -3.22 & \\
QE water-cation SCCS & -1 & -5.60 \\
QE water SoftCS & -3.14 & -5.69 \\
Born $\left(r_{\mathrm{Cl}^{-}}=1.81 \AA, r_{\mathrm{Na}^{+}}=0.97 \AA\right)$ & -3.93 & -7.33 \\
\hline
\end{tabular}

Table II. The more sophisticated implicit solvent calculations give sơmewhat smaller differences compared to the above Born value, i.e., SMD model (Gaussian16 calculation) gives the $\Delta \Delta G_{\text {solv }}$ difference of $1.3 \mathrm{eV}$ for $\mathrm{Cl}^{-}$, whereas QUANTUM ESPRESSO gives the difference of $1.4 \pm 0.1 \mathrm{eV}$, depending on the specific implicit solvent method. These are sizable differences and represent an important contribution to the SAM hindrance of the penetration of $\mathrm{Cl}^{-}$.

It should be noted, though, that in the current context, the relevant difference is the difference in the real solvation free energies ${ }^{31}$ between the two phases, because as the ion moves from one phase to the other it needs to make work against the dipole barrier that develops at the water/SAM interface. The two solvation free energy differences are related by:

$$
\Delta \Delta G_{\mathrm{solv}}^{\mathrm{real}}=\Delta \Delta G_{\mathrm{solv}}+q_{\mathrm{ion}} \chi
$$

where $q_{\text {ion }}$ is the charge of the ion and $\chi$ is the dipole potential at the interface between the two dielectrics. For halide anions the real solvation free energies $\left(\Delta G_{\text {solv }}^{\text {real }}\right)$ for the transfer of ion from vacuum into aqueous solution are by 0.2 to $0.3 \mathrm{eV}$ more endergonic than the solvation free energies $\left(\Delta G_{\text {solv }}\right){ }^{31}$

In this study dielectrics are modeled implicitly, hence the dipole potential $\chi$ at the interface between the two dielectrics cannot be properly described-for liquids this dipole potential by and large arises due to preferential molecular orientations at the interface-hence in the following we will not make any distinction between $\Delta \Delta G_{\text {solv }}^{\text {real }}$ and $\Delta \Delta G_{\text {solv }}$ and, for simplicity, only the $\Delta \Delta G_{\text {solv }}$ designation will be used.

\section{Results}

As shown above, to understand the penetration of $\mathrm{Cl}^{-}$from water into $\mathrm{SAM} / \mathrm{metal}$ substrate the knowledge of the following four important aspects is required: (i) solvation, (ii) inter-ion electrostatics, (iii) depolarization effects, and (iv) 3D PBC artifacts. It is worth emphasizing that the last two effects can be avoided by proper modeling. By using appropriate models as to avoid 
Table II

Comparison of calculated solvation free energies of $\mathrm{Cl}^{-}$and $\mathrm{Na}^{+}$ions in water and $\mathrm{SAM}$ as given by various methods. The difference in $\Delta G_{\text {solv }}$ between the two solvents are also given, $\Delta \Delta G_{\text {solv }}=\Delta G_{\text {solv }}(\mathrm{SAM})-\Delta G_{\text {solv }}$ (water). Rows labeled as " $\mathrm{Cl}^{-}+\mathrm{Na}^{+}$" report the sum of $\mathrm{Cl}^{-}$and $\mathrm{Na}^{+} \Delta \Delta G_{\text {solv }}$ values.

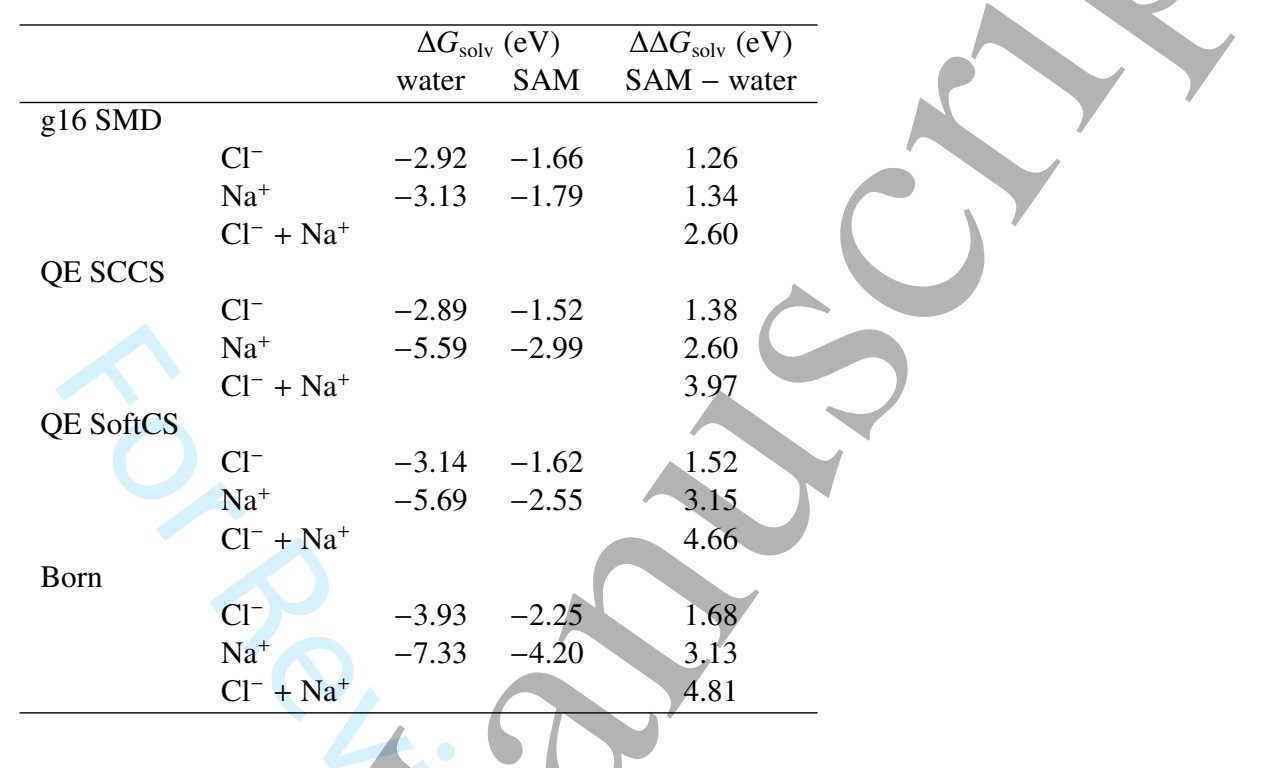

effects (iii) and (iv), we performed several sets of DFT calculations with QE, where only $\mathrm{Cl}^{-}$and $\mathrm{Na}^{+}$ions were treated explicitly, whereas water, SAM, and metal slab were described implicitly as described in the Technical section. With aid of the theory presented above we will analyze below the QE calculated data as to disentangle the inter-ion electrostatics and solvation effects; by the term "inter-ion electrostatics" we refer only to the electrostatic interactions between ions, whereas the electrostatic interaction of an ion with a dielectric is treated under solvation effects. As for the inter-ion electrostatic part, the $U_{11}$ and $U_{12}$ components of the explicit Eq. [14] are too complicated to be used for fitting the QE calculated data, whereas Eq. [15] for $U^{\text {approx }}(z)$ is too approximate, because it does not describe the "soft" transition of the interaction energy at the interface (cf. Fig. 6). In analogy to Eq. [B.3] in the Appendix, where the lattice sum is approximated by the integral, i.e.:

$$
\sum_{\boldsymbol{R}} \frac{q}{R_{z}}=\sum_{\boldsymbol{R}} \frac{q}{\sqrt{R^{2}+z^{2}}} \approx 2 \pi \sigma \int \frac{R}{R_{z}} \mathrm{~d} R=2 \pi \sigma \sqrt{R^{2}+z^{2}}+\text { const. }
$$

we will use the $U(z) \propto \sqrt{R^{2}+z^{2}}$ ansatz. In particular, the interaction energy $U(z)$ will be fitted to:

$$
U(z) \approx U_{0}+ \begin{cases}+\frac{2 \pi q^{2}}{A \varepsilon_{\mathrm{sam}}}\left(\sqrt{\alpha^{2}+z^{2}}-|\alpha|\right) & \text { for } z \leq 0 \\ -\frac{2 \pi q^{2}}{A \varepsilon_{\text {water }}} z & \text { for } z>0,\end{cases}
$$

where the water/SAM interface is at $z=0, \varepsilon_{\mathrm{sam}}$ and $\varepsilon_{\mathrm{water}}$ are the permittivities of SAM and water, respectively, $\alpha$ is the fitting parameter, and $U_{0}$ is a reference energy at $z=0$ (for convenience it can be set to 0$)$. Note that in the above equation the water part of $U(z)$ was simplified, because the $\partial U(z) / \partial z$ slope in water is so much smaller than that in SAM that using the simplified linear expression is adequate. 

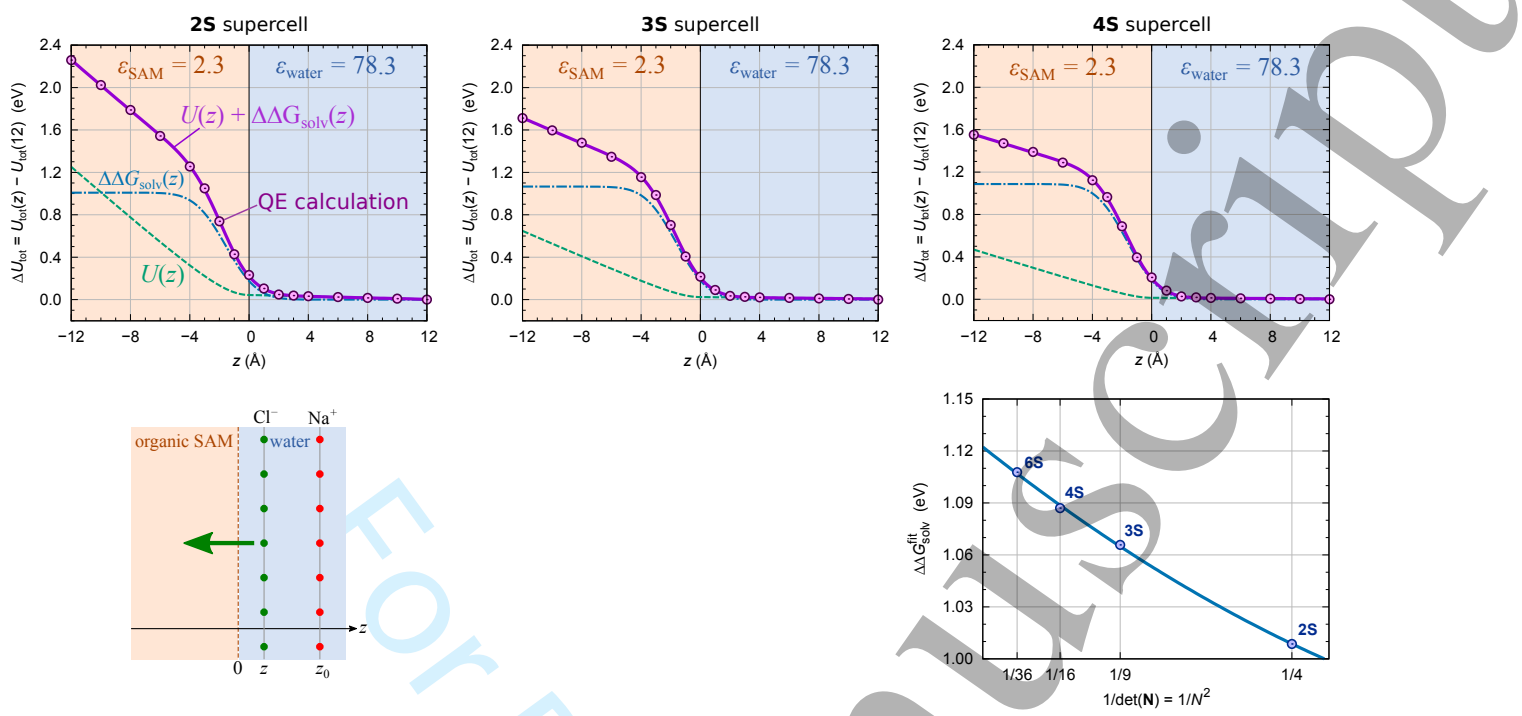

Figure 10. Top-row: QE calculated interaction energies (points) for parallel infinite layers of $\mathrm{Cl}^{-}$and $\mathrm{Na}^{+}$ions near the $\mathrm{SAM}$ /water interface, with dielectrics modeled implicitly, for $\mathbf{2 S}, \mathbf{3 S}$, and $\mathbf{4 S}$ supercells; the setup is shown schematically on the bottom-left (the $\mathrm{Na}^{+}$layer is held fixed in water, whereas the $\mathrm{Cl}^{-}$layer is moved inward to the left). Total QE calculated interaction energies (points) are fitted with the $U_{\text {tot }}(z)$ function of Eq. [2,5] (purple curve) and its components, $U(z)$ (dashed green curve) and $\Delta \Delta G_{\text {solv }}(z)$ (dot-dashed blue curve), are also shown. Bottom-right: difference of solvation free energies between SAM and water phases, $\Delta \Delta G_{\text {solv }}^{\mathrm{wls}}$ of Eq. [23], versus the inverse of the supercell size as obtained from fitting the $\mathrm{QE}$ data (as a measure of the size of the supercell $\operatorname{det}(\mathbf{N})=N^{2}$ is used).

As for solvation effects, it is worth noting that an ion is not a point object but has a finite size and, also, in QE the dielectric interface is modeled as having some spread. Hence, the transition in solvation free energy from water to SAM phases is smooth. For this reason the change of solvation free energy will be modeled as:

$$
\Delta \Delta G_{\mathrm{solv}}(z)=\frac{1}{2} \operatorname{erfc}\left(\frac{z+z_{\mathrm{w} \mid \mathrm{s}}}{r_{\mathrm{w} \mid \mathrm{s}}}\right) \Delta \Delta G_{\mathrm{solv}}^{\mathrm{w} \mid \mathrm{s}},
$$

where erfc () is the complementary error function, $z_{\mathrm{w} \mid \mathrm{s}}$ and $r_{\mathrm{w} \mid \mathrm{s}}$ are fitting parameters (label "w|s" stands for "water|SAM"), and $\Delta \Delta G_{\text {solv }}^{\mathrm{wl}}$ is, in principle, the real solvation free energy difference between SAM and water phases (cf. Eq. [20]):

$$
\Delta \Delta G_{\mathrm{solv}}^{\mathrm{wls}} \approx \Delta \Delta G_{\mathrm{solv}}^{\mathrm{real}}=\Delta G_{\mathrm{solv}}(\mathrm{SAM})-\Delta G_{\mathrm{solv}}(\text { water })+q_{\mathrm{ion}} \chi,
$$

although the distinction between real free energy and free energy of solvation is not really relevant here, because $\Delta \Delta G_{\text {solv }}^{\mathrm{w} / \mathrm{s}}$ is a fitting parameter and, moreover, the implicit dielectric models cannot properly describe the dipole potential $\chi$.

The total interaction energy, obtained from QE calculations, is therefore fitted by the $U_{\text {tot }}(z)$ function, defined as:

$$
U_{\text {tot }}(z)=U(z)+\Delta \Delta G_{\text {solv }}(z)
$$

where for $U(z)$ the simplified version of Eq. [22] is used. 
A simpler case: SAM/water system.-

Before analyzing the results for the whole metal/SAM/water system, let us first analyze a simpler case, that is, a layer of $\mathrm{Cl}^{-}$ions near the SAM/water interface with the layer of $\mathrm{Na}^{+}$counter-ions held fixed in water above the $\mathrm{Cl}^{-}$ layer. Fig. 10 shows the results for the $\mathbf{2 S}, \mathbf{3 S}$, and $\mathbf{4 S}$ supercells (6S supercell was also considered). These large supercells prevent the depolarization effects (in the considered $z$ range) and allow to disentangle the solvation effects from the inter-ion electrostatic repulsion as evident from the perfect fit of QE calculated data points by Eq. [25] (purple curve). The dependence of the interaction energy on the z-coordinate of the $\mathrm{Cl}^{-}$layer can be described as follows. For all considered supercells, the interaction energy appears nearly constant in water, due to its large permittivity. As the $\mathrm{Cl}^{-}$ions pass from water into the SAM, the energy first raises quickly with decreasing $z$ and the corresponding $\partial U_{\text {tot }}(z) / \partial z$ slope is roughly independent of the supercell size: this increase in energy is mainly due to inferior solvation of $\mathrm{Cl}^{-}$ions in SAM compared to water (i.e., the $\Delta \Delta G_{\text {solv }}(z)$ component). Upon further penetration of $\mathrm{Cl}^{-}$ions into SAM, for $z \lesssim-4 \AA$, the slope of $\partial U_{\text {tot }}(z) / \partial z$ becomes less steep and also dependent on the supercell size, i.e., the smaller is the supercell the steeper is the slope. This dependence is due to electrostatic interactions between ions (i.e., the $U(z)$ component). In particular, the attraction between the $\mathrm{Cl}^{-}$and $\mathrm{Na}^{+}$layers diminishes linearly as the distance between the two layers increases with the $\partial U(z) / \partial z$ slope being proportional to $A^{-1}$, where $A$ is the supercell area. The decomposition of $U_{\text {tot }}(z)$ to the $\Delta \Delta G_{\text {solv }}(z)$ and $U(z)$ components yields about $1.1 \mathrm{eV}$ for the difference in solvation free energy of $\mathrm{Cl}^{-}$between SAM and water (note from the bottom-right panel of Fig. 10 that the so estimated $\Delta \Delta G_{\text {solv }}^{\mathrm{w} / \mathrm{s}}$ slightly increases with the supercell size, but the dependence is weak and the value extrapolated to the infinitely large supercell is $1.12 \mathrm{eV}$ ). This value is somewhat smaller than the difference calculated from calculations of standalone ions (cf. Table II).

The whole metal/SAM/water system. - Before commenting on the interaction energy of the penetrating $\mathrm{Cl}^{-}$layer from water into the $\mathrm{SAM} /$ metal substrate, let us remind that metal is modeled as implicit dielectric with huge permittivity constant of a few thousands ${ }^{\dagger}$ and that such a model is representative for liquid metal, e.g., mercury at room temperature. For this reason $\mathrm{Cl}^{-}$ions can easily penetrate into such metal-model without any elastic penalty or Pauli repulsion that would be present in a solid metal.

In the metal/SAM/water system, there are two interfaces-metal/SAM and SAM/waterhence Eqs. [22] and [23] for $U(z)$ and $\Delta \Delta G_{\text {solv }}(z)$ should be augmented to account for the additional metal/SAM interface. In compliance with the previous subsection, the SAM/water interface is kept at $z=0$, hence the metal/SAM interface is at $z=-w_{\text {sam }}$, where $w_{\text {sam }}$ is the SAM width (thickness). The corresponding augmented equation for $U(z)$ is:

$$
U(z) \approx U_{0}+ \begin{cases}\frac{2 \pi q^{2}}{A \varepsilon_{\mathrm{sam}}}\left(2 \sqrt{\alpha^{2}+\left(\frac{w_{\mathrm{sam}}}{2}\right)^{2}}-2|\alpha|\right) & \text { for } z<-w_{\mathrm{sam}} \\ \frac{2 \pi q^{2}}{A \varepsilon_{\mathrm{sam}}}\left(2 \sqrt{\alpha^{2}+\left(\frac{w_{\mathrm{sam}}}{2}\right)^{2}}-\sqrt{\alpha^{2}+\left(z+w_{\mathrm{sam}}\right)^{2}}-|\alpha|\right) & \text { for } z \in\left[-w_{\mathrm{sam}},-\frac{w_{\mathrm{sam}}}{2}\right] \\ \frac{2 \pi q^{2}}{A \varepsilon_{\mathrm{sam}}}\left(\sqrt{\alpha^{2}+z^{2}}-|\alpha|\right) & \text { for } z \in\left(-\frac{w_{\mathrm{sam}}}{2}, 0\right) \\ -\frac{2 \pi q^{2}}{A \varepsilon_{\text {wate }}} z & \text { for } z \geq 0,\end{cases}
$$

We arbitrarily used $\varepsilon_{\text {metal }}=2666$. With this value the $\varepsilon_{\text {metal }} / \varepsilon_{\text {water }}$ ratio is the same as the $\varepsilon_{\text {water }} / \varepsilon_{\text {sam }}$ ratio. 
2S supercell
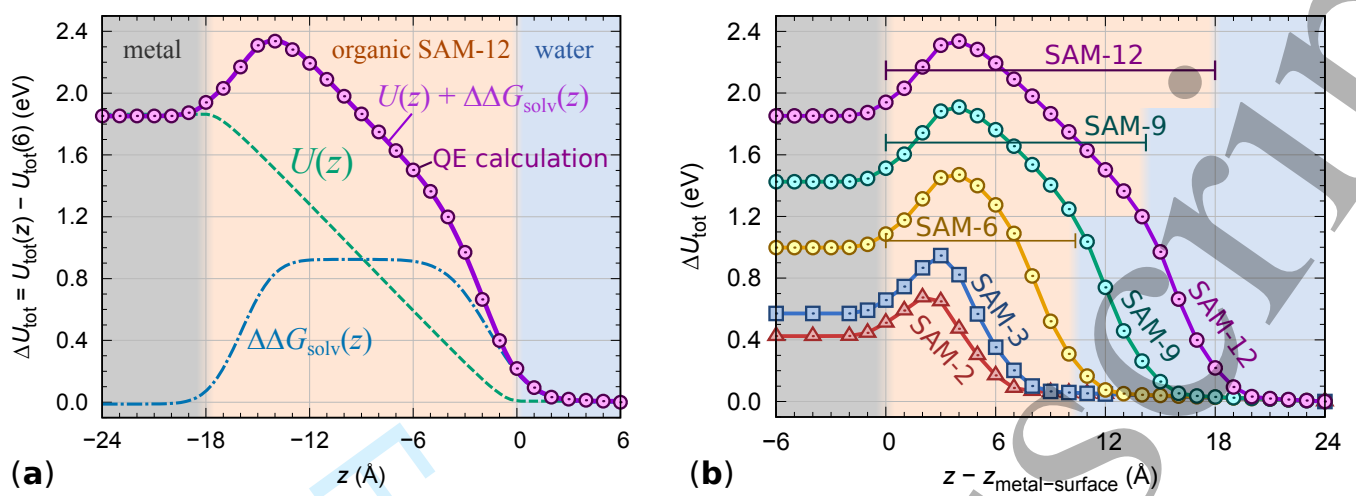

4S supercell
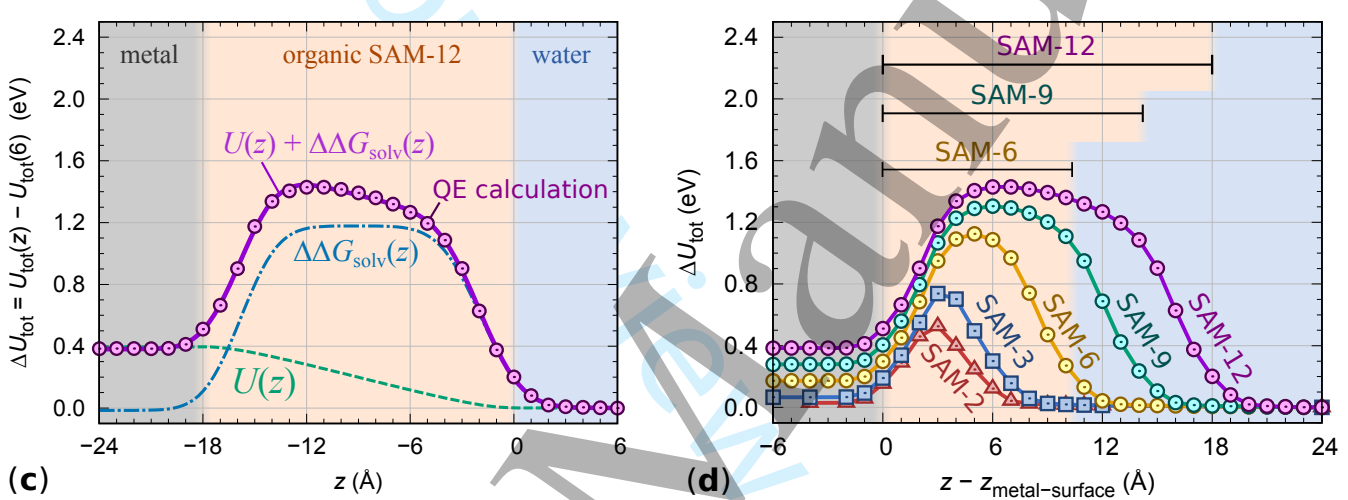

Figure 11. QE calculated interaction energies (points) for the penetration of $\mathrm{Cl}^{-}$layer from water into the implicit $\mathrm{SAM} /$ metal systems for the $\mathbf{2 S}$ (top row) and $\mathbf{4 S}$ (bottom row) supercells; the layer of $\mathrm{Na}^{+}$is held fixed in water above the $\mathrm{Cl}^{-}$layer. (a,c) QE calculated interaction energies of the metal/SAM-12/water system (points); these data points are fitted by the $U_{\text {tot }}(z)$ function of Eq. [25] and its components, $\Delta \Delta G_{\text {solv }}(z)$ of Eq. [26] and $U(z)$ of Eq. [27], are also shown. (b,d) QE calculated interaction energies for the metal/SAM- $x$ /water systems for $x=2,3,6,9$, and 12 . Thicknesses of the SAM-12, SAM-9, and SAM-6 layers are also indicated.

where $\alpha$ is a fitting parameter. Note that the $U(z)$ term for $z<-w_{\text {sam }}$ is set to a constant, which implies that the metal is considered to be a perfect conductor. The corresponding augmented equation for $\Delta \Delta G_{\text {solv }}(z)$ is:

$$
\Delta \Delta G_{\mathrm{solv}}(z)=\frac{1}{2}\left[\operatorname{erfc}\left(\frac{z+z_{\mathrm{w} \mid \mathrm{s}}}{r_{\mathrm{w} \mid \mathrm{s}}}\right) \Delta \Delta G_{\mathrm{solv}}^{\mathrm{w} \mid \mathrm{s}}-\operatorname{erfc}\left(\frac{z+z_{\mathrm{m} \mid \mathrm{s}}+w_{\mathrm{sam}}}{r_{\mathrm{m} \mid \mathrm{s}}}\right) \Delta \Delta G_{\mathrm{solv}}^{\mathrm{m} \mid \mathrm{s}}\right]
$$

where the labels $\mathrm{w} \mid \mathrm{s}$ and $\mathrm{m} / \mathrm{s}$ indicate the water/SAM and metal/SAM interfaces, respectively, and $z_{\mathrm{w} \mid \mathrm{s}}, z_{\mathrm{m} \mid \mathrm{s}}, r_{\mathrm{w} \mid \mathrm{s}}, r_{\mathrm{m} \mid \mathrm{s}}$, and $\Delta \Delta G_{\mathrm{solv}}^{\mathrm{wls}}$ are fitting parameters, ${ }^{\ddagger}$ whereas $\Delta \Delta G_{\mathrm{solv}}^{\mathrm{m} / \mathrm{s}}$ is calculated from

\footnotetext{
The number of parameters can be reduced by setting $z_{\mathrm{w} \mid \mathrm{s}}=z_{\mathrm{m} \mid \mathrm{s}}$ and $r_{\mathrm{w} \mid \mathrm{s}}=r_{\mathrm{m} \mid \mathrm{s}}$.
} 
$\Delta \Delta G_{\mathrm{solv}}^{\mathrm{wls}}$ by utilizing the Born model:

$$
\Delta \Delta G_{\mathrm{solv}}^{\mathrm{m} / \mathrm{s}}=\left(1-\frac{1}{\varepsilon_{\mathrm{water}}}\right)^{-1} \Delta \Delta G_{\mathrm{solv}}^{\mathrm{w} / \mathrm{s}}
$$

The results for the penetration of the $\mathrm{Cl}^{-}$layer from water into the $\mathrm{SAM} /$ metal substrate is shown in Fig. 11 for the $\mathbf{2 S}$ and $\mathbf{4 S}$ supercells. The plots on the left side of the figure display the results for the SAM-12/metal substrate, where the QE calculated data points are fitted by the $U_{\text {tot }}(z)=U(z)+\Delta \Delta G_{\text {solv }}(z)$ function with the two components defined by Eqs. [26] and [27]. The excellent fit provides confidence that the presented physical picture is sound and that the $U(z)$ and $\Delta \Delta G_{\text {solv }}(z)$ components are reliably determined. The value of $\Delta \Delta G_{\text {solv }}^{\mathrm{wl}}$, extrapolated to zero coverage analogously to that in the the bottom-right of Fig. 10, is $1.26 \mathrm{eV}$, which is somewhat smaller than the difference calculated from calculations of standalone ions (cf. Table II). The interaction energy profile around the SAM/water interface is similar as for the SAM/wateronly system (Fig. 10), that is, in water the interaction energy is nearly constant and then it rises steeply as the $\mathrm{Cl}^{-}$ions enter into SAM due to the inferior solvation therein. After about $4 \AA$ into SAM, the energy profile becomes less steep with the energy rising linearly with the slope of $-2 \pi q^{2} /\left(\varepsilon_{\text {sam }} A\right)$ up until the $\mathrm{Cl}^{-}$comes to within about $4 \AA$ from the metal surface (note that the slope for the larger $\mathbf{4 S}$ supercell is four times smaller compared to that of the smaller $\mathbf{2 S}$ supercell). From this point on, the energy decreases due to superior solvation of $\mathrm{Cl}^{-}$ions in the "liquid metal" and becomes constant once the $\mathrm{Cl}^{-}$ions enter the metal. The right-part of Fig. 11 shows the QE calculated data points for the metal/SAM- $x$ /water systems for $x=2,3,6,9$, and 12 . It can be seen that the barrier for the penetration of $\mathrm{Cl}^{-}$ions toward the metal surface decreases from SAM-12 to SAM-2. From SAM-12 to SAM-6 this is mainly due decreasing thickness of SAM and consequently lower $U(z)$ electrostatic repulsion contribution. Note that this contribution decreases as the supercell size increases (or surface density of $\mathrm{Cl}^{-}$ions decreases), i.e., $U(z) \propto A^{-1} \propto \sigma$, and becomes vanishing for very small coverage. In contrast, the $\Delta \Delta G_{\text {solv }}(z)$ component is largely independent on the SAM thickness provided that SAM is sufficiently thick. Namely, the bottom-right plot of Fig. 11 suggests that, due to spatial extent of the $\Delta \Delta G_{\text {solv }}(z)$ transition, the $\Delta \Delta G_{\text {solv }}(z)$ barrier will start decreasing for SAM thicknesses smaller than about $10 \AA$ and indeed this effect is clearly seen for SAM-3 and SAM-2, whose thickness is about 6.6 and $5.3 \AA$, respectively.

In Fig. 11 the metal substrate is considered to be directly in contact with the SAM. However, metal surfaces are oxidized and metal-oxides have much smaller permittivity (for example, the relative static permittivity of $\mathrm{Al}_{2} \mathrm{O}_{3}$ is about 10). ${ }^{32}$ For this reason, Fig. 12 compares the implicit metal/SAM-6/water system with two implicit oxide/SAM-6/water systems with the permittivity values of 5 and 10 for the oxide dielectrics. It can be seen that the barrier for the penetration of $\mathrm{Cl}^{-}$is not much affected by the considerably smaller permittivity of oxides; the differences mainly emerge after the barrier (that is, for $z-z_{\text {surf }} \lesssim 4 \AA$ ) and are related to a smaller ionic solvation in the implicit oxide compared to that in the metal and much weaker electrostatic screening of the oxide, which results in the more steep slope of the energy curve once the ions enter the oxide.

Fig. 11 suggests that irrespective of the details of the implicit substrate (either metal or oxide), some attractive interactions between $\mathrm{Cl}^{-}$and the oxidized surface are to be expected. Such attractive interactions are indeed plausible if the oxidized surface is hydroxylated, however, the implicit description of the oxide is definitely too simplistic to describe this situation (implicit dielectric with an extraordinarily large permittivity can be at best an approximate model of the

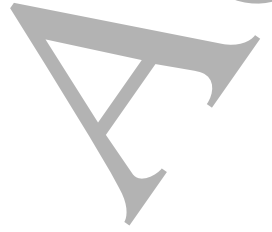




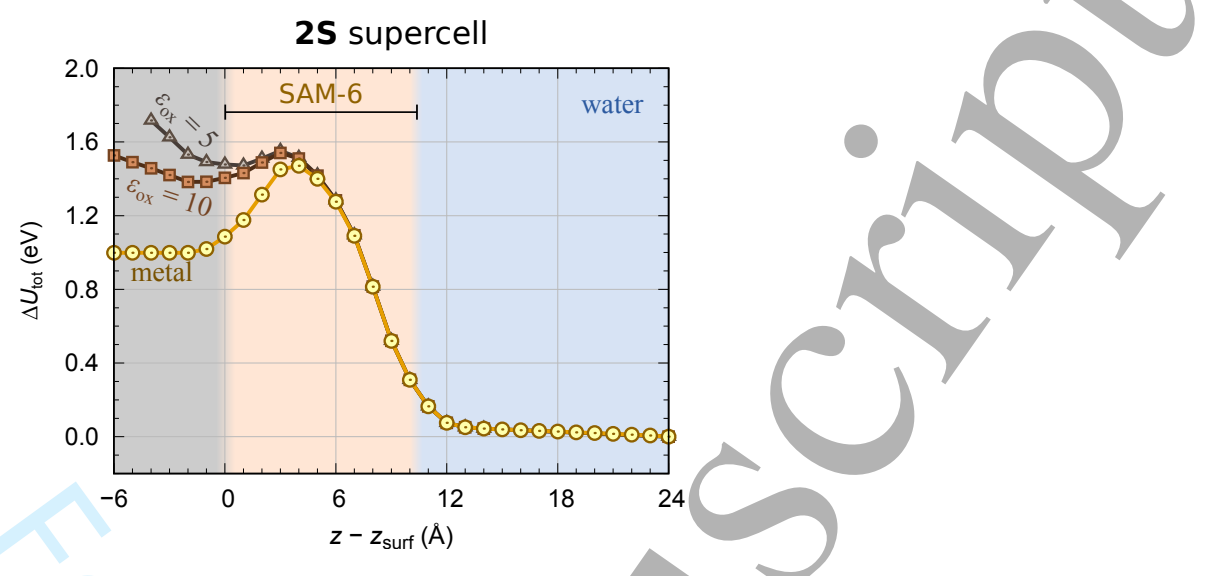

Figure 12. Similar as in Fig. 11b, but here the energy profile of the implicit metal/SAM-6/water system is compared to those for two implicit oxide/SAM-6/water systems, with the oxide permittivity values of $\varepsilon_{\mathrm{ox}}=5$ and 10 . The position of metal/SAM and oxide/SAM interfaces is at $z=z_{\text {surf }}$.

liquid mercury and even there implicit description cannot describe chemical interactions). In the next publication ${ }^{20}$ we will address this issue with the explicit atomistic DFT modeling, but here only a very simple $2 \mathrm{D}$ point-charge model is utilized instead. In particular, the structure of the top layer of the hydroxylated oxide-film of the $\mathrm{AlOOH} / \mathrm{Al}_{2} \mathrm{O}_{3} / \mathrm{Al}(111)$ model of Fig. 3 is taken in a $\mathbf{2 S}$ supercell configuration and $\mathrm{Na}, \mathrm{Cl}, \mathrm{H}, \mathrm{O}$, and $\mathrm{Al}$ ions are modeled as $+1,-1,+1,-2$, and +3 point-charges, respectively (the structure of this $2 \mathrm{D}$ model-with one $\mathrm{Cl}^{-}$and $\mathrm{Na}^{+}$per supercell-is shown on the right side of Fig. 13). The model is charge-neutral and contains 48 $\mathrm{H}, 60 \mathrm{O}$, and $24 \mathrm{Al}$ point-charge ions per $\mathbf{2 S}$ supercell. The electrostatic Coulomb energy (per supercell) of this point-charge model is calculated as:

$$
\begin{aligned}
U & =\frac{1}{2 \varepsilon} \sum_{\boldsymbol{R}=0}^{\infty} \sum_{i, j}^{N} \frac{q_{i} q_{j}}{\left|\boldsymbol{R}+\boldsymbol{\tau}_{j}-\boldsymbol{\tau}_{i}\right|}\left(1-\delta_{i, j} \delta_{R, 0}\right) \\
& \simeq \frac{1}{2 \varepsilon}\left(\sum_{\boldsymbol{R}=0}^{\boldsymbol{R}_{\mathrm{cut}}} \sum_{i, j}^{N} \frac{q_{i} q_{j}}{\left|\boldsymbol{R}+\boldsymbol{\tau}_{j}-\boldsymbol{\tau}_{i}\right|}\left(1-\delta_{i, j} \delta_{R, 0}\right)+\frac{2 \pi}{A} \int_{R_{\mathrm{cut}}}^{\infty} \sum_{i, j}^{N} \frac{q_{i} q_{j} R}{\sqrt{R^{2}+\left(z_{j}-z_{i}\right)^{2}}} \mathrm{~d} R\right) \\
& \simeq \frac{1}{2 \varepsilon} \sum_{\boldsymbol{R}=0}^{\boldsymbol{R}_{\mathrm{cut}}} \sum_{i, j}^{N} \frac{q_{i} q_{j}}{\left|\boldsymbol{R}+\boldsymbol{\tau}_{j}-\boldsymbol{\tau}_{i}\right|}\left(1-\delta_{i, j} \delta_{R, 0}\right)-\frac{\pi}{\varepsilon A} \sum_{i, j}^{N} q_{i} q_{j} \sqrt{R_{\mathrm{cut}}^{2}+\left(z_{j}-z_{i}\right)^{2}},
\end{aligned}
$$

where $A$ is the area of the supercell, $z_{i}$ is the $z$-component of the position of an ion $i, \tau_{i}=$ $\left(x_{i}, y_{i}, z_{i}\right)$, and the $\left(1-\delta_{i, j} \delta_{R, 0}\right)$ term is used to omit the ion self-interaction. $R_{\text {cut }}$ is the cutoff radius up to which the lattice sums are evaluated explicitly and beyond it they are approximated by the integral. At first sight, the result of this integral appears to be linear in $R_{\text {cut }}$ and thus divergent at the $R_{\text {cut }} \rightarrow \infty$ limit. This, however, is not the case, because due to charge neutrality this term is proportional to $R_{\text {cut }}^{-1}$ and thus tends to zero as $R_{\text {cut }} \rightarrow \infty$ (see the Supporting Information of Ref. 33). For the permittivity $\varepsilon$, the value of 2.3 of SAM is taken. The results of this model for $\mathrm{Cl}$ ions approaching the hydroxylated oxide surface are shown in Fig. 13, where the average position of $\mathrm{Al}$ ions is taken as the surface plane $\left(z_{\text {surf }}=0\right)$. The interaction energy profile indeed mimics those shown in Figs. 11 and 12. In particular, it can be seen that for $z-z_{\text {surf }} \gtrsim 6 \AA$ the 

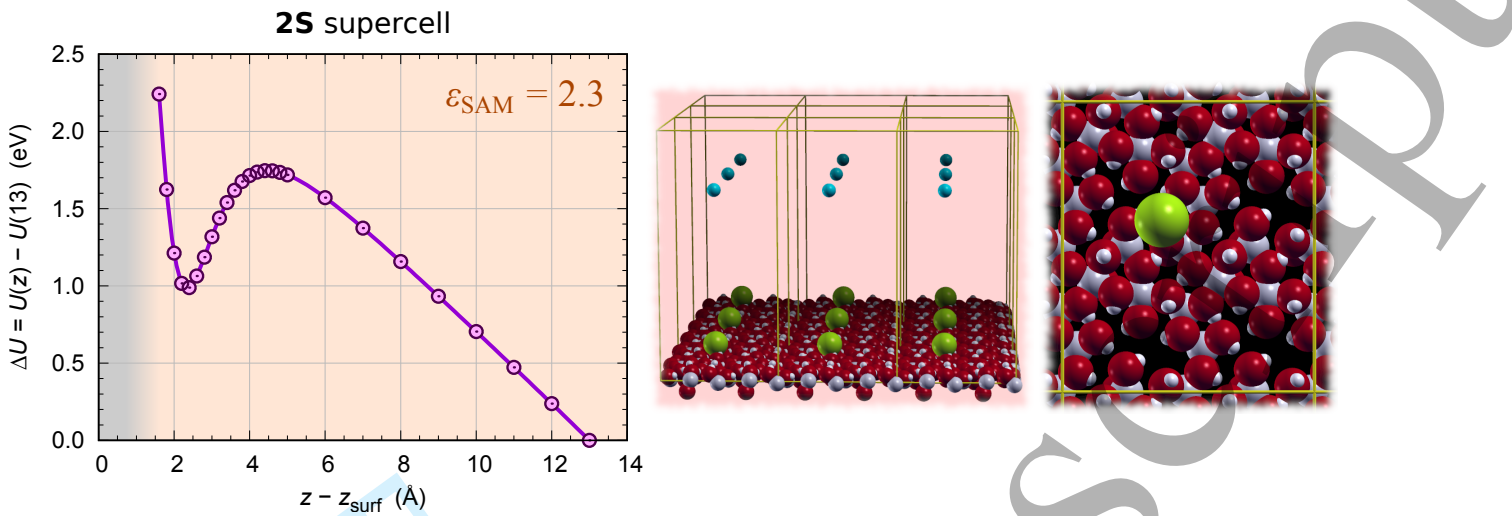

Figure 13. Electrostatic energy of the $2 \mathrm{D}$ PBC point-charge model immersed in implicit SAM dielectric $\left(\varepsilon_{\mathrm{SAM}}=2.3\right)$ as a function of the height of $\mathrm{Cl}^{-}$ions above the surface $\left(z-z_{\text {surf }}\right)$. The average height of $\mathrm{Al}$ ions is taken as the surface plane, $z_{\text {surf }}=0$. For the reference zero energy, the energy for $\mathrm{Cl}^{-}$at $z-z_{\text {surf }}=13 \AA$ is arbitrarily used. The structure of the 2D PBC point-charge model is shown on the right (perspective view of several supercells and top view of a single supercell); the model consists of the top layer of the hydroxylated oxide-film of the $\mathrm{AlOOH} / \mathrm{Al}_{2} \mathrm{O}_{3} / \mathrm{Al}(111)$ model of Fig. 3 in a $2 \mathrm{~S}$ supercell configuration, where the $\mathrm{H}, \mathrm{O}$, and $\mathrm{Al}$ ions are represented by $+1,-2$, and +3 point-charges, respectively.

energy profile is linear (note that here only SAM without water above it is considered), but below about $5 \AA$ the energy starts decreasing and reaches a minimum at about $2.5 \AA$ from the surface. This simple model thus reproduces the suggestion of the implicit models (cf. Fig. 12) that $\mathrm{Cl}^{-}$ ions can interact attractively with the surface after the "penetration" barrier is passed.

Penetration of a charge-neutral layer of $\mathrm{Na}^{+} / \mathrm{Cl}^{-}$ions from water into the SAM/metal substrate.- $\mathrm{Na}^{+}$ions solvate much stronger than $\mathrm{Cl}^{-}$(see Table I), hence from this point of view their presence in SAM seems unlikely. But is it viable that $\mathrm{Na}^{+}$cation penetrates together with the $\mathrm{Cl}^{-}$anion into SAM? In general, $\mathrm{Na}^{+}$concomitantly identified with $\mathrm{Cl}^{-}$is associated with the presence of residuals from the electrolyte. For example, chloride entry into passive films (not covered with inhibitors) have been studied with surface science tools, especially by X-ray photoelectron spectroscopy (XPS). It is known that $\mathrm{Cl}^{-}$may penetrate into the passive films and that the $\mathrm{Na}^{+}$counter-ions are not present therein. ${ }^{34} \mathrm{Can}$, however, $\mathrm{Na}^{+}$ions be present in organic SAM? To the best of our knowledge, very few experiments have been performed to measure the amount of chloride adsorption in the presence of an inhibitor layer. From the available data, no $\mathrm{Na}^{+}$counter-ions were identified when $\mathrm{Cl}^{-}$anions are present in the inhibitor layer. ${ }^{35-37} \mathrm{To}$ further illuminate the question of whether $\mathrm{Na}^{+}$ions can be present in inhibitor layers, the results of the corresponding model calculations are presented below.

Fig. 14 shows the $\mathrm{QE}$ calculated energy profiles for the penetration of a charge-neutral layer, consisting of $\mathrm{Na}^{+} / \mathrm{Cl}^{-}$ions from water into the SAM/metal substrate. Three different $\mathrm{Na}^{+} / \mathrm{Cl}^{-}$ configurations are considered: a "uniform" layer of $\mathrm{Na}^{+} / \mathrm{Cl}^{-}$ions with the $\mathrm{Na}^{+}$and $\mathrm{Cl}^{-}$coordinates of $(0,0, z)$ and $(a / 2, b / 2, z)$, respectively, and two different layers of $\mathrm{Na}^{+} / \mathrm{Cl}^{-}$ion-pairs, with the ion-pairs oriented along either $z$ or $x$ directions (the corresponding schemes are shown Fig. 14). The main observations are the following: due to the charge-neutrality of the layers the interaction energies are flat in each phase (far enough from the interfaces) and the energy position in a given phase is determined by two effects: (i) the electrostatic attraction between $\mathrm{Na}^{+}$and $\mathrm{Cl}^{-}$ ions in the layer (notice that this contribution is more attractive in SAM than in water, because 
1S supercell
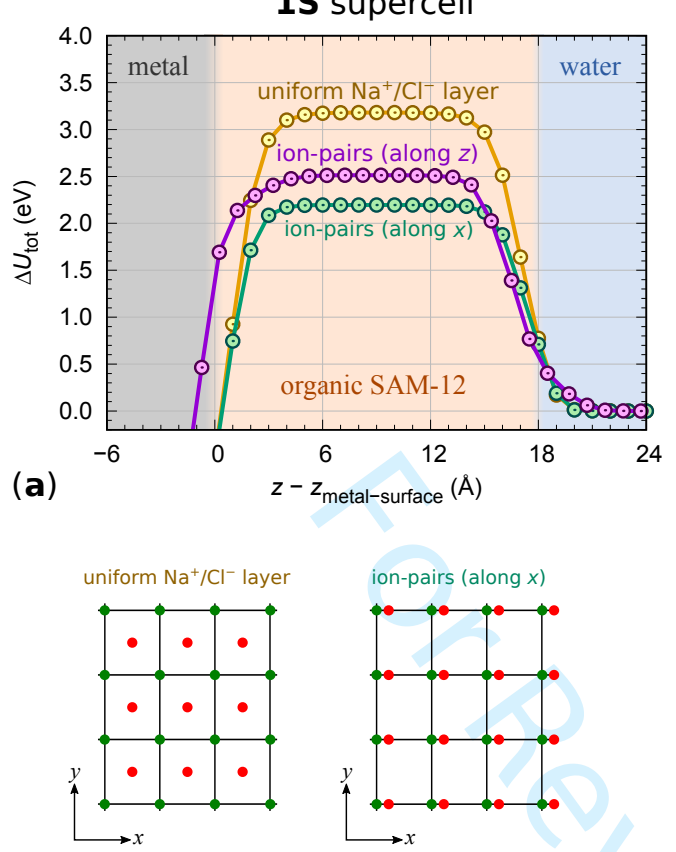

$z$ ion-pairs (along $z$ )

$$
:-1:-
$$

(b)
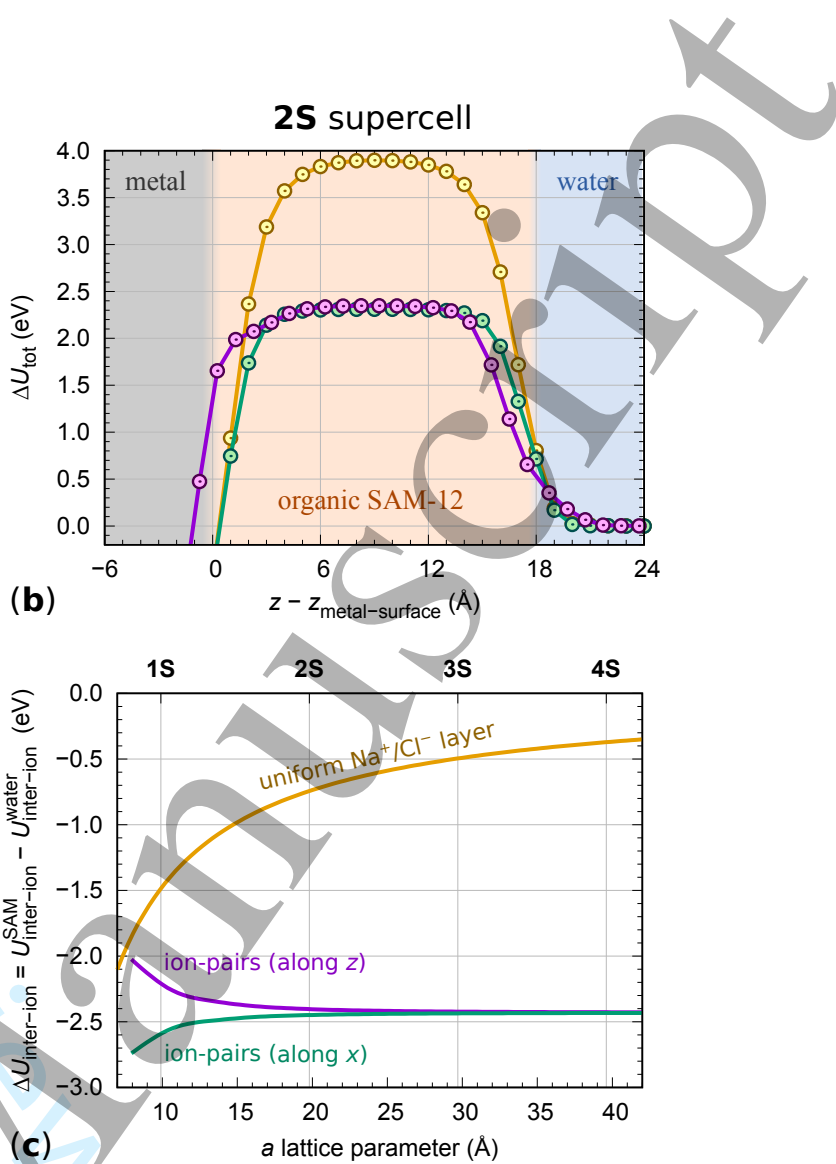

Figure 14. (a,b) QE calculated interaction energies for the penetration of charge-neutral $\mathrm{Na}^{+} / \mathrm{Cl}^{-}$layer from water into the implicit SAM-12/metal system for the 1S and 2S supercells. Three different variants are considered (the corresponding schematics are shown at the bottom left), i.e., a "uniform" layer of $\mathrm{Na}^{+} / \mathrm{Cl}^{-}$ions with coordinates of $(0,0, z)$ and $(a / 2, b / 2, z)$ and two different layers of $\mathrm{Na}^{+} / \mathrm{Cl}^{-}$ion-pairs, with the ion-pairs oriented along either $z$ or $x$ directions. For the layer of $z$-oriented ion-pairs, the ion-pair barycenter is taken as the reference $z$ position. (c) Difference between plain electrostatic attraction between ions in SAM and water, $\Delta U_{\text {inter-ion }}=U_{\text {inter-ion }}^{\text {SAM }}-U_{\text {inter-ion }}^{\text {water }}$, as a function of the $a$ lattice parameter for the $\mathbf{S}$ type lattices for the three considered configurations of ions (QE optimized $\mathrm{Na}-\mathrm{Cl}$ distance for

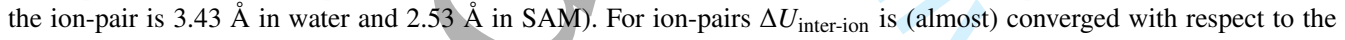
supercell size already for the $\mathbf{2 S}$ supercell (ion-pair can be seen as a dipole), whereas for the uniform configuration the interactions are far more long-ranged.

water much better screens electrostatic interactions) and (ii) the difference in solvation free energy of the ions between the two phases. The solvation of ions in water is much stronger than in SAM and this contribution wins over the electrostatic attraction between ions in the layer, which is why the energy is much higher in SAM than in water. In particular, for the uniform layer of $\mathrm{Na}^{+} / \mathrm{Cl}^{-}$ions, compatible with the $1 \mathrm{~S}$ supercell, the electrostatic attraction is about $1.5 \mathrm{eV}$ stronger in $\mathrm{SAM}$ than in water, but the solvation of $\mathrm{Na}^{+}$and $\mathrm{Cl}^{-}$ions is stronger by about $4.7 \mathrm{eV}$ per ion-pair in water than in SAM, hence the difference is about $3.2 \mathrm{eV}$. This difference coincides with the QE calculated difference between the stability of the uniform $\mathrm{Na}^{+} / \mathrm{Cl}^{-}$layer in SAM and water (Fig. 14a). For the larger $\mathbf{2 S}$ supercell the electrostatic attraction between ions is reduced, because ions are farther apart from each other. The difference between inter-ion attraction in SAM and water thus diminishes to about $0.8 \mathrm{eV}$ (Fig. 14c) and the uniform $\mathrm{Na}^{+} / \mathrm{Cl}^{-}$layer is by 
about $4.7-0.8=3.9 \mathrm{eV}$ less stable in SAM than in water (Fig. 14b).

Fig. 14 further reveals that in SAM the two considered layers of ion-pairs are more stable than the uniform $\mathrm{Na}^{+} / \mathrm{Cl}^{-}$layer, mainly due to additional "intra-molecular" electrostatic stabilization within each ion-pair. For the smaller $\mathbf{1 S}$ supercell the layer of ion-pairs oriented along $x$-direction is by about $0.3 \mathrm{eV}$ more stable that the layer of $z$-oriented ion-pairs. Ion-pairs can be seen as dipoles and it is obvious from the bottom-left part of Fig. 14 that the $x$-oriented dipoles are more stable than the $z$-oriented dipoles (for the latter the inter-dipole interactions are exclusively repulsive, whereas for the $x$-oriented dipoles the inter-dipole interactions are attractive along the $x$ direction and repulsive along the $y$ direction). It is worth noting from Fig. $14 \mathrm{c}$ that for ion-pairs the dipole-dipole interactions converge much faster with respect to the supercell size-they are (almost) converged already for the $\mathbf{2 S}$ supercell-than those of the uniform $\mathrm{Na}^{+} / \mathrm{Cl}^{-}$layer (the latter tend to zero as the supercell size goes to infinity).

The results presented in Fig. 14 suggest that $\mathrm{Na}^{+}$ion would penetrate SAM together with $\mathrm{Cl}^{-}$ ion in the form of an ion-pair. However, by comparing the penetration barriers in Fig. 14 to those in Fig. 11 it is evident that the barrier for the penetration of the $\mathrm{Na}^{+} / \mathrm{Cl}^{-}$ion-pair is by about 1 $\mathrm{eV}$ higher than that for the penetration of the $\mathrm{Cl}^{-}$ion, which suggests that the penetration of $\mathrm{Cl}^{-}$ alone is favorable.

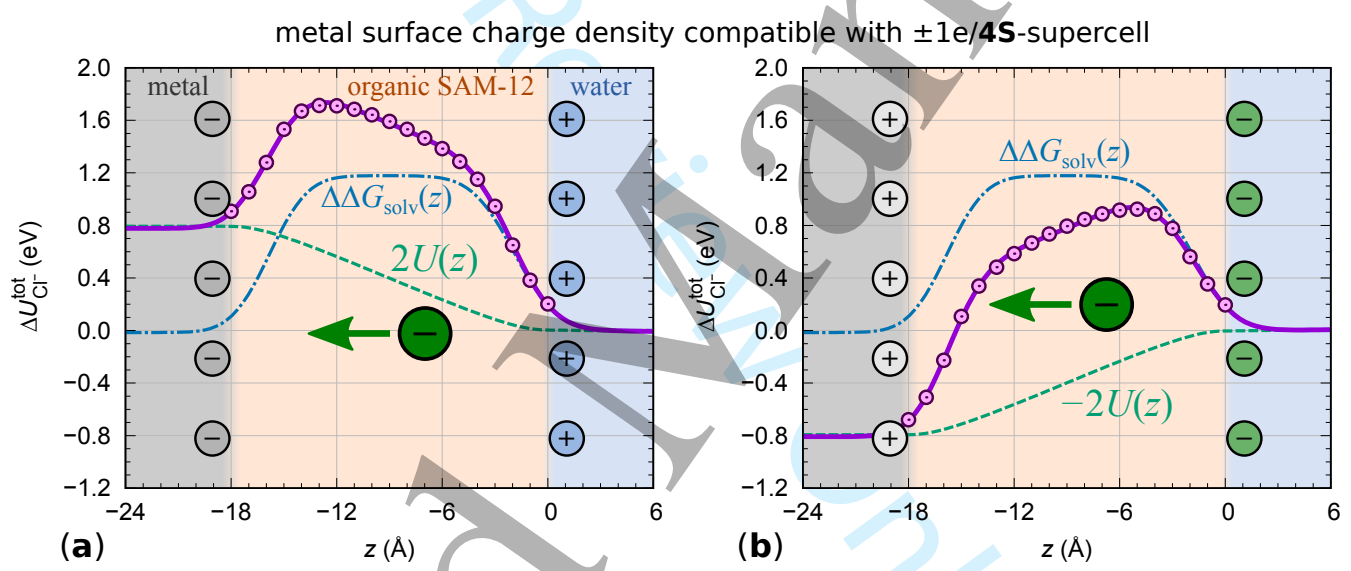

Figure 15. Model calculation of the potential energy, $U_{\mathrm{Cl}^{-}}^{\text {tot }}(z)$ of Eq. [30], for the penetration of a single $\mathrm{Cl}^{-}$ion from water into the SAM-12/metal system, according to the model described in the text, for (a) negatively and (b) positively charged metal surface with the magnitude of the surface charge density equivalent to 1 e/4S-supercell $=0.074 \mathrm{e} / \mathrm{nm}^{2}$. The electrostatic $U(z)$ and solvation $\Delta \Delta G_{\text {solv }}(z)$ components are also shown. Data points correspond to QE calculated data, $\mathrm{QE}\left(z_{i}\right)$ from Fig. 11c, that were augmented for the current purpose, in particular, in (a) the data points correspond to $\mathrm{QE}\left(z_{i}\right)+U\left(z_{i}\right)$ and in (b) to $\mathrm{QE}\left(z_{i}\right)-3 U\left(z_{i}\right)$.

Dependence of the penetration barrier on the electrode potential: a simple Helmholtz-like double-layer picture. - The repulsive electrostatic component $U(z)$, shown in Fig. 11, develops because the whole $\mathrm{Cl}^{-}$layer moves away from the layer of $\mathrm{Na}^{+}$counter-ions. While on the one hand this is an artifact due to lateral periodic boundary conditions-in reality it is extraordinarily more likely that $\mathrm{Cl}^{-}$ions penetrate SAM individually-on the other hand, it can be seen to mimic the effect of the electrode potential. To elaborate on this point, let us imagine a simple picture of the Helmholtz-like electrochemical double-layer, where the potential drop entirely happens in SAM and SAM itself is impermeable to ions (actually almost impermeable, because we will 
consider the penetration of individual ions). The system thus consists of metal covered by SAM and $\mathrm{Na}^{+}$and $\mathrm{Cl}^{-}$ions in aqueous solvent.

For electrode potentials that are negative with respect to the potential of zero charge, the metal surface is negatively charged, hence the $\mathrm{Na}^{+}$ions will be attracted by the surface and will make a counter-layer on top of SAM (recall that SAM is impermeable) and so an electric double-layer is formed. Now imagine that a single $\mathrm{Cl}^{-}$ion penetrates the SAM (Fig. 15a). This situation is very similar to that shown in Fig. 11, but with the important difference that here the electrostatic component in the SAM is given by $2 U(z)$ and is therefore twice steeper than in the case considered above, where the whole $\mathrm{Cl}^{-}$layer moves away from the $\mathrm{Na}^{+}$layer toward the charge-neutral metal surface. The reason for the twice steeper slope is the following. As the $\mathrm{Cl}^{-}$ ion moves away from the $\mathrm{Na}^{+}$layer it needs to overcome attraction that pulls $\mathrm{Cl}^{-}$in the opposite direction and this costs $2 \pi q \sigma / \varepsilon_{\text {sam }}$ per unit of $z$, where $\sigma$ is the magnitude of the surface charge density of ions and $q$ is the magnitude of the individual charge (in the case of $\mathrm{Na}^{+}$and $\mathrm{Cl}^{-}$ions, $q=q_{\mathrm{Na}^{+}}=-q_{\mathrm{Cl}^{-}}=1$ and $\sigma=A^{-1}$ ). But $\mathrm{Cl}^{-}$also moves toward the negatively charged metal surface, hence it needs to overcome repulsion which also costs $2 \pi q \sigma / \varepsilon_{\text {sam }}$ per unit of $z$. Hence the two contributions sum and the electrostatic component displays the $-4 \pi q \sigma / \varepsilon_{\text {sam }}$ slope (recall that the minus sign is due to $\mathrm{Cl}^{-}$moving in the negative $z$ direction).

For electrode potentials that are positive with respect to the potential of zero charge the situation is the opposite. The metal surface is positively charged, hence the $\mathrm{Cl}^{-}$ions will make a counter-layer on top of SAM thus forming an electric double-layer (Fig. 15b). In this case the penetrating $\mathrm{Cl}^{-}$ion is attracted by the positively charged metal surfaces and pushed toward it by the repulsive interaction from the layer of $\mathrm{Cl}^{-}$ions on top of SAM, hence the electrostatic component in SAM is given by $-2 U(z),{ }^{\S}$ where the slope depends on the surface charge density at the metal surface and is proportional to $4 \pi q \sigma / \varepsilon_{\mathrm{sam}}$.

These insights can be used to estimate the dependence of the $\mathrm{Cl}^{-}$penetration barrier on the electrode potential. For convenience let us denote the total interaction energy by $U_{\mathrm{Cl}^{-}}^{\text {tot }}$ and the electrostatic component by $U_{\mathrm{Cl}^{-}}(z)= \pm 2 U(z)$, where the subscript " $\mathrm{Cl}^{-}$" is used as a mnemonic that this energy corresponds to the penetration of a single $\mathrm{Cl}^{-}$ion toward the charged metal surface. In analogy to Eq. [25], the total potential energy can therefore be written as:

$$
U_{\mathrm{Cl}^{-}}^{\mathrm{tot}}(z)=\Delta \Delta G_{\mathrm{solv}}(z)+U_{\mathrm{Cl}^{-}}(z)= \begin{cases}\Delta \Delta G_{\mathrm{solv}}(z)+2 U(z) & \text { for } E<E_{\mathrm{pzc}} \\ \Delta \Delta G_{\mathrm{solv}}(z)-2 U(z) & \text { for } E>E_{\mathrm{pzc}}\end{cases}
$$

where the $\Delta \Delta G_{\text {solv }}(z)$ and $U(z)$ components are given by Eqs. [26] and [27], respectively (pedantically, this equality holds only for $z \in \mathrm{SAM}$, see below). $E$ stands for the electrode potential and $E_{\mathrm{pzc}}$ for the electrode potential of zero charge (PZC). It should be noted that $\Delta \Delta G_{\text {solv }}(z)$ and $U(z)$ functions are known as they have been obtained by fitting the data of Fig. 11.

It is useful to generalize Eq. [30] and to explicitly indicate its dependence on the ion charge $\left(q_{\text {ion }}\right)$ and the metal surface charge density $\left(\sigma_{\mathrm{m}}\right)$, i.e.:

$\S$ Note that the electrostatic component is constant outside SAM, taking one value for $z<-w_{\text {sam }}$ and another one at $U_{\text {iop }}^{\text {tot }}\left(z ; q_{\text {ion }}, \sigma_{\mathrm{m}}\right)=\Delta \Delta G_{\text {solv }}\left(z ; q_{\text {ion }}\right)+U_{\text {ion }}\left(z ; q_{\text {ion }}, \sigma_{\mathrm{m}}\right)$,

$>0$, cf. Eq. [32].

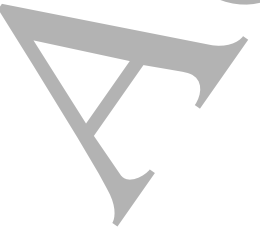


where $U_{\text {ion }}\left(z ; q_{\text {ion }}, \sigma_{\mathrm{m}}\right)$ is given by:

$$
U_{\text {ion }}\left(z ; q_{\text {ion }}, \sigma_{\mathrm{m}}\right)=U_{0}+ \begin{cases}\frac{4 \pi q_{\mathrm{ion}} \sigma_{\mathrm{m}}}{\varepsilon_{\mathrm{sam}}}\left(2 \sqrt{\alpha^{2}+\left(\frac{w_{\mathrm{sam}}}{2}\right)^{2}}-2|\alpha|\right) & \text { for } z<-w_{\mathrm{sam}} \\ \frac{4 \pi q_{\mathrm{ion}} \sigma_{\mathrm{m}}}{\varepsilon_{\mathrm{sam}}}\left(2 \sqrt{\alpha^{2}+\left(\frac{w_{\mathrm{sam}}}{2}\right)^{2}}-\sqrt{\alpha^{2}+\left(z+w_{\mathrm{sam}}\right)^{2}}-|\alpha|\right) & \text { for } z \in\left[-w_{\mathrm{sam}},-\frac{w_{\mathrm{sam}}}{2}\right] \\ \frac{4 \pi q_{\mathrm{ion}} \sigma_{\mathrm{m}}}{\varepsilon_{\mathrm{sam}}}\left(\sqrt{\alpha^{2}+z^{2}}-|\alpha|\right) & \text { for } z \in\left(-\frac{w_{\mathrm{sam}}}{2}, 0\right) \\ 0 & \text { for } z \geq 0\end{cases}
$$

Note that $U_{\text {ion }}$ takes constant values for $z<-w_{\text {sam }}$ and $z>0$; this is where $U_{\text {ion }}$ differs from $\pm 2 U(z)$. The reason is that the two layers of charges are located at $z=-w_{\text {sam }}$ and $z=0$ and outside of the double-layer there is no double-layer induced electric-field (furthermore water and particularly metal efficiently screen electrostatic interactions).

The activation barrier for the $\mathrm{Cl}^{-}$penetration $\left(U_{\mathrm{Cl}^{-}}^{\text {act }}\right)$ is given by the maximum of the $U_{\text {ion }}^{\text {tot }}(z)$ function in SAM, which obviously depends on the metal surface charge density $\sigma_{\mathrm{m}}$, cf. Eq. [31], hence also $U_{\mathrm{Cl}^{-}}^{\text {act }}$ depends on $\sigma_{\mathrm{m}}$, i.e.:

$$
U_{\mathrm{Cl}^{-}}^{\text {act }}\left(\sigma_{\mathrm{m}}\right)=\max _{z \in \mathrm{SAM}} U_{\mathrm{ion}}^{\mathrm{tot}}\left(z ; q \mathrm{Cl}^{-}, \sigma_{\mathrm{m}}\right)
$$

The maximum of $U_{\text {ion }}^{\text {tot }}\left(z ; q_{\mathrm{Cl}^{-}}, \sigma_{\mathrm{m}}\right)$ for $/ \mathrm{a}$ given $\sigma_{\mathrm{m}}$ is given by the condition $\partial U_{\text {ion }}^{\text {tot }}\left(z ; q_{\mathrm{Cl}^{-}}, \sigma_{\mathrm{m}}\right) / \partial z=0$, which gives:

$$
\frac{\partial U_{\mathrm{ion}}}{\partial z}+\frac{\exp \left(-\left(z+z_{\mathrm{w} \mid \mathrm{s}}\right)^{2} / r_{\mathrm{w} \mid \mathrm{s}}^{2}\right)}{r_{\mathrm{w} \mid \mathrm{s}} \sqrt{\pi}} \Delta \Delta G_{\mathrm{solv}}^{\mathrm{w} \mid \mathrm{s}}-\frac{\exp \left(-\left(z+z_{\mathrm{m} \mid \mathrm{s}}\right)^{2} / r_{\mathrm{m} \mid \mathrm{s}}^{2}\right)}{r_{\mathrm{m} \mid \mathrm{s}} \sqrt{\pi}} \Delta \Delta G_{\mathrm{solv}}^{\mathrm{m} \mid \mathrm{s}}=0
$$

and

$$
\frac{\partial U_{\mathrm{ion}}}{\partial z}= \begin{cases}-\frac{4 \pi q_{\mathrm{ion}} \sigma_{\mathrm{m}}}{\varepsilon_{\mathrm{sam}}} \frac{z+w_{\mathrm{sam}}}{\sqrt{\alpha^{2}+\left(z+w_{\mathrm{sam}}\right)^{2}}} & \text { for } z \in\left[-w_{\mathrm{sam}},-\frac{w_{\mathrm{sam}}}{2}\right] \\ +\frac{4 \pi q_{\mathrm{ion}} \sigma_{\mathrm{m}}}{\varepsilon_{\mathrm{sam}}} \frac{z}{\sqrt{\alpha^{2}+z^{2}}} & \text { for } z \in\left(-\frac{w_{\mathrm{sam}}}{2}, 0\right), \\ 0 & \text { otherwise. }\end{cases}
$$

This is a messy equation and, therefore, Eq. [33] was solved numerically.

To complete the picture we need to link the metal surface charge density with the electrode potential and to this end the aforementioned simple Helmholtz-like electrochemical double-layer model, where the potential drop $(\Delta E)$ entirely happens in SAM, is utilized. Within this model the electrode potential relative to $\mathrm{PZC}, E-E_{\mathrm{pzc}}$, is given by this potential drop, which according to the setup of Fig. 15, can be obtained from the electrostatic $U_{\text {ion }}(z)$ contribution at $z=-w_{\text {sam }}$, i.e.:

$$
\Delta E=E-E_{\mathrm{pzc}}=\frac{U_{\text {ion }}\left(-w_{\mathrm{sam}}\right)}{q_{\text {ion }}} .
$$

Here the surface dipole potential $\chi$ at the SAM/water interface is neglected, because the currently utilized implicit calculations cannot describe it. According to Eqs. [32] and [36] $\Delta E$ can be written as the following function of $\sigma_{\mathrm{m}}$ :

$$
\Delta E\left(\sigma_{\mathrm{m}}\right)=E\left(\sigma_{\mathrm{m}}\right)-E(0)=\frac{4 \pi \sigma_{\mathrm{m}}}{\varepsilon_{\mathrm{sam}}}\left(\sqrt{4 \alpha^{2}+w_{\mathrm{sam}}^{2}}-2|\alpha|\right)
$$


which, if $\alpha$ is small, can be approximated by:

$$
\Delta E\left(\sigma_{\mathrm{m}}\right) \approx \frac{4 \pi \sigma_{\mathrm{m}}}{\varepsilon_{\mathrm{sam}}}\left(w_{\mathrm{sam}}-2|\alpha|\right) \approx \frac{4 \pi \sigma_{\mathrm{m}}}{\varepsilon_{\mathrm{sam}}} w_{\mathrm{sam}} .
$$

Note that Eqs. [37] and [38] are written in Hartree atomic units, hence the electrode potential has a unit of Hartree/e $(1$ Hartree/e $=27.21 \mathrm{~V})$, where "e" is the unit charge. These equations reveal that, in addition to $\sigma_{\mathrm{m}}, \Delta E$ depends also on the SAM thickness $w_{\text {sam }}$ and this implies that at a given electrode potential the magnitude of the surface charge density is larger for thin SAMs (low $w_{\text {sam }}$ ) than for thick SAMs (high $w_{\text {sam }}$ ), because $\Delta E \propto \sigma_{\mathrm{m}} w_{\text {sam }}$.

Let us now consider also the penetration of individual $\mathrm{Na}^{+} / \mathrm{Cl}^{-}$ion-pairs. At PZC the energy profile is as shown in Fig. 14b, but for electrode potentials away from PZC the ion-pair can orient $^{\text {II }}$ along the electric field $(\mathcal{E})$ as to lower the energy by $-\mathcal{E} \cdot \boldsymbol{\mu}=-\mathcal{E} \mu$, where $\boldsymbol{\mu}$ is the dipole moment of the $\mathrm{Na}^{+} / \mathrm{Cl}^{-}$ion-pair. Note that the electric field in SAM is assumed to have only the surface normal component, $\mathcal{E}=(0,0, \mathcal{E})$, hence also $\boldsymbol{\mu}=(0,0, \mu)$. For the positively charged metal surface the $\mathrm{Na}^{+} / \mathrm{Cl}^{-}$ion-pair orients with $\mathrm{Cl}^{-}$toward the metal surface and vice-versa for the negatively charged metal surface. Within the current model, the electric field in SAM can be written as $\mathcal{E}=-q_{\text {ion }}^{-1} \partial U_{\text {ion }} / \partial z$, where $\partial U_{\text {ion }} / \partial z$ is given by Eq. [35], but for practical purposes this can be approximated by the following function of $\sigma_{\mathrm{m}}$ :

$$
\mathcal{E}\left(\sigma_{\mathrm{m}}\right) \approx-\frac{4 \pi \sigma_{\mathrm{m}}}{\varepsilon_{\mathrm{sam}}}
$$

For not too strong electric fields, the barrier for the penetration of the $\mathrm{Na}^{+} / \mathrm{Cl}^{-}$ion-pair from water through SAM toward the metal surface can be approximated as:

$$
U_{\mathrm{Na}^{+} / \mathrm{Cl}^{-}}^{\mathrm{act}}\left(\sigma_{\mathrm{m}}\right) \approx \Delta \Delta G_{\mathrm{solv}, \mathrm{Na}^{+} / \mathrm{Cl}^{-}}^{\mathrm{wls}}-\left|\frac{4 \pi \sigma_{\mathrm{m}}}{\varepsilon_{\mathrm{sam}}} \mu\right|,
$$

where $\Delta \Delta G_{\mathrm{solv}, \mathrm{Na}^{+} / \mathrm{Cl}^{-}}^{\mathrm{w}}$ is the difference of the solvation free energy of the $\mathrm{Na}^{+} / \mathrm{Cl}^{-}$ion-pair between SAM and water phases, i.e., $\Delta \Delta G_{\text {solv, } \mathrm{Na}^{+} / \mathrm{Cl}^{-}}^{\mathrm{wls}}$ is analogous to $\Delta \Delta G_{\text {solv }}^{\mathrm{wls}}$ in Eq. [27] but parameterized for the $\mathrm{Na}^{+} / \mathrm{Cl}^{-}$ion-pair instead of $\mathrm{Cl}^{-}$. As stated above the $\mathrm{Na}^{+} / \mathrm{Cl}^{-}$ion-pair is assumed to align with the electric field, hence the corresponding term is written such that it always diminishes the activation barrier.

Eq. [40] suggests that for strong enough electric fields (or surface charge densities), the barrier would drop to zero. However, the electric field takes the full effect only when the ion-pair is fully in SAM, but the change in solvation from water to SAM kicks in prior to that. This results in the non-vanishing penetration barrier even for electric fields that are stronger than those for which Eq. [40] predicts no barrier. For this reason the dependence of the penetration activation energy $U_{\mathrm{Na}^{+} / \mathrm{Cl}^{-}}^{\text {act }}$ on the surface charge density $\sigma_{\mathrm{m}}$ was scrutinized by performing QE calculations (see Appendix) and the so-obtained data were then fitted by a quadratic function of $\sigma_{\mathrm{m}}$ :

$$
U_{\mathrm{Na}^{+} / \mathrm{Cl}^{-}}^{\mathrm{act}}\left(\sigma_{\mathrm{m}}\right)=\Delta \Delta G_{\mathrm{solv}, \mathrm{Na}^{+} / \mathrm{Cl}^{-}}^{\mathrm{w}}-a_{1}\left|\sigma_{\mathrm{m}}\right|+a_{2} \sigma_{\mathrm{m}}^{2},
$$

where $a_{1}$ and $a_{2}$ are the fitting parameters. For further details on dependence of $U_{\mathrm{Na}^{+} / \mathrm{Cl}^{-}}^{\text {act }}$ on $\sigma_{\mathrm{m}}$, see Appendix.

IINote that currently SAM is described by implicit dielectric continuum, hence SAM itself does not prefer any orientation of the ion-pair nor is there any elastic penalty for reorientation. 

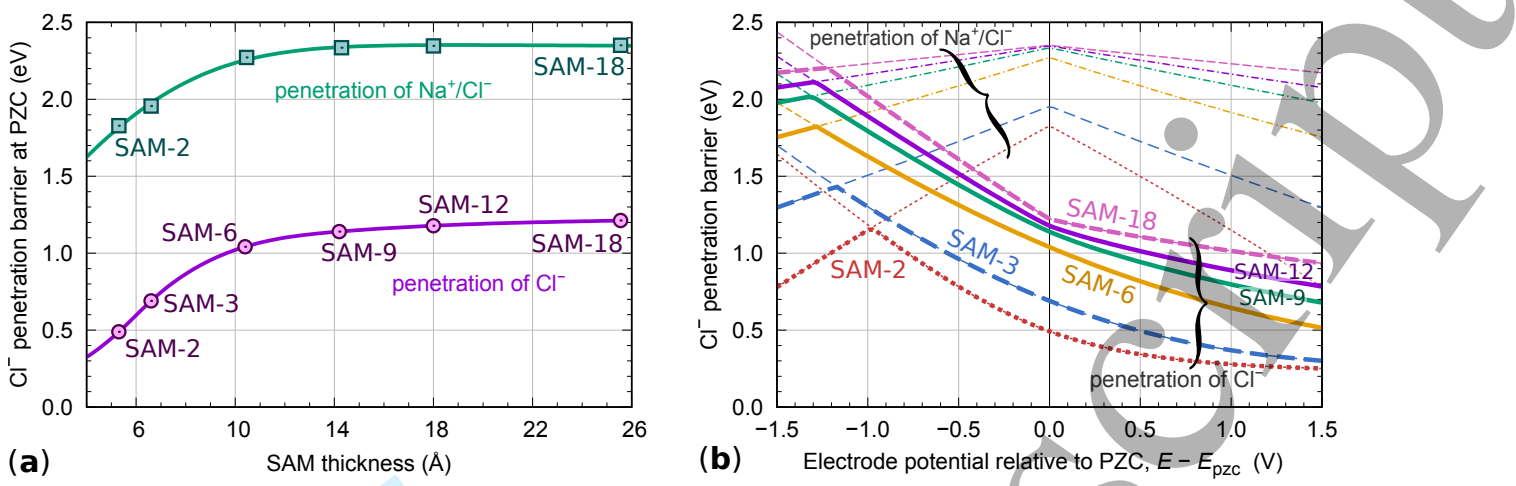

Figure 16. (a) Activation barriers for the penetration of standalone $\mathrm{Cl}^{-}$(purple) and of the $\mathrm{Na}^{+} / \mathrm{Cl}^{-}$ion-pair (cyan) at PZC as functions of the SAM thickness. (b) Activation barriers for the penetration into SAM- $x$ with $x \in\{2,3,6,9,12,18\}$ as functions of the electrode potential (relative to PZC). The activation barriers for both the standalone $\mathrm{Cl}^{-}$ion and the $\mathrm{Na}^{+} / \mathrm{Cl}^{-}$ion-pair are considered, where the former are calculated by Eq. [33] and the latter by Eq. [41].

In the above equations the electrode potential, Eq. [37], and the $\mathrm{Cl}^{-}$penetration barriereither for the standalone $\mathrm{Cl}^{-}$ion, Eq. [33], or for the $\mathrm{Na}^{+} / \mathrm{Cl}^{-}$ion-pair, Eq. [41]—are expressed as functions of the metal surface charge density, hence the dependence of penetration barrier on the electrode potential can be plotted parametrically (the parameter being the metal surface charge density). The corresponding results are shown in Fig. 16b, whereas Fig. 16a plots the $\mathrm{Cl}^{-}$and $\mathrm{Na}^{+} / \mathrm{Cl}^{-}$penetration barriers at PZC as functions of the SAM thickness. At PZC the barrier is due to the $\Delta \Delta G_{\text {solv }}(z)$ component and the figure shows that for thin SAMs the barrier increases with the SAM thickness but then it "saturates" for sufficiently thick SAMs (say, for $w_{\text {sam }} \gtrsim 10 \AA$ ); this is the trend already discussed above in conjunction with Fig. 11 . As for the dependence of the $\mathrm{Cl}^{-}$penetration barrier on the electrode potential, Fig. 16b reveals, as expected, that for standalone $\mathrm{Cl}^{-}$the barrier decreases with increasing electrode potential (for thick enough SAMs, the slope changes abruptly at PZC). In contrast, the barrier for the penetration of the $\mathrm{Na}^{+} / \mathrm{Cl}^{-}$ion-pair decreases in both directions, i.e., for $E<E_{\mathrm{pzc}}$ and for $E>E_{\mathrm{pzc}}$. But the $\Delta \Delta G_{\text {solv }}$ component is much higher for the $\mathrm{Na}^{+} / \mathrm{Cl}^{-}$ion-pair than for standalone $\mathrm{Cl}^{-}$, hence only at sufficiently negative potentials the $\mathrm{Na}^{+} / \mathrm{Cl}^{-}$barrier becomes smaller than the $\mathrm{Cl}^{-}$barrier (for thick SAMs this happens at highly negative potentials). This implies that the penetration of $\mathrm{Na}^{+}$ions (more specifically $\mathrm{Na}^{+} / \mathrm{Cl}^{-}$ion-pairs) into SAM can become viable at highly negative electrode potentials, particularly for thin SAMs.

Finally, for proper understanding of Fig. 16, the assumptions and approximations that were used to derive the results should be clearly stated. These are: (i) it is assumed that CA- $x$ molecules fully cover the metal surface thus forming a complete SAM irrespectively of the size of the CA molecules (in contrast, it was shown by experiments that only long-chain CA molecules completely cover Al surfaces), ${ }^{6}$ (ii) surface is fully covered by SAM at all electrode potentials, (iii) electrode potential (relative to PZC) is entirely given by the potential drop in SAM, (iv) surface dipole potential at SAM/water interface is neglected, (v) water, SAM, and metal are represented implicitly by dielectric continuum, hence the model is unable to capture the "penalty" for local deformation of SAM during the $\mathrm{Cl}^{-}$or $\mathrm{Na}^{+} / \mathrm{Cl}^{-}$penetration, and (vi) implicitly calculated solvation free energies of ions are only approximate (the error is most likely larger for $\mathrm{Na}^{+}$than for $\mathrm{Cl}^{-}$). On this basis, the results of Fig. 16 should be understood qualitatively, i.e., they are good enough to ascertain trends, but particular quantitative values may be susceptible to 
significant errors.

\section{Conclusions}

In summary, we analyzed the mechanism by which organic self-assembled-monolayers hinder the penetration of $\mathrm{Cl}^{-}$ions from aqueous solution toward the metal substrate. To this end, we developed a simple scheme that qualitatively describes the activation barrier for $\mathrm{Cl}^{-}$penetration into SAM as a function of the electrode potential. The scheme predicts that the activation barrier decreases as the electrode potential increases, as would be intuitively expected. However, the decrease of the activation barrier is rather gradual for electrode potential that are positive with respect to PZC and for thick SAMs the barrier remains sizable even at rather positive potentials. This may be one among the reasons why dense and sufficiently thick SAMs efficiently inhibit corrosion.

By utilizing a simple model where metal substrate, organic layer, and aqueous solvent are described implicitly by dielectric continuum slabs, we identified and analyzed two important effects by which SAMs hinder the penetration of $\mathrm{Cl}^{-}$ions toward the metal substrate. The first effect is due to inferior solvation of ions in organic layer compared to that in aqueous solvent and the estimated difference is larger than $1 \mathrm{eV}$ for SAMs thicker than about $10 \AA$, whereas for thinner SAMs this difference decreases with decreasing SAM thickness. The second effect is due to the electric field at the electrochemical interface and the extent by which it affects the penetration of $\mathrm{Cl}^{-}$depends on the electrode potential and on the thickness of the organic layer. It is worth emphasizing that the presented model is not specific to $\mathrm{Cl}^{-}$ions, but can be applied also to anodic dissolution of metal, i.e., diffusion of metal cations from the metal substrate through SAM toward the solvent, irrespectively of whether metal dissolves in the form of bare cations or coordinated to ligands in a metal-ligand complexes, provided that the complexes are charged. Indeed, the model is based on electrostatic arguments and is therefore applicable to any charged species.

The penetration of $\mathrm{Na}^{+}$counter-ions into organic layer was also addressed and calculations indicate that due to their stronger solvation the penetration into organic layer is inferior to that of $\mathrm{Cl}^{-}$. Energetically the most favorable way for $\mathrm{Na}^{+}$to penetrate into SAM is in the form of $\mathrm{Na}^{+} / \mathrm{Cl}^{-}$ion-pairs, but it is inferior to penetration of $\mathrm{Cl}^{-}$alone, unless the electrode potential becomes sufficiently negative (for thick SAMs the required value would be "extreme").

While the developed scheme for estimating the activation barrier for $\mathrm{Cl}^{-}$penetration into SAM as a function of the electrode potential is based on several assumptions and approximations, we believe that it is sufficiently good to ascertain trends, although particular quantitative values are likely susceptible to significant errors. One among the effects that the current implicit calculations cannot describe is the energy penalty associated with the local deformation of the organic layer during the $\mathrm{Cl}^{-}$penetration and this effect will be addressed in our next publication ${ }^{20}$ by means of explicit DFT calculations.

Finally, we also elucidated and discussed various aspects that are relevant for modeling charged ions near surfaces with slab models that utilize periodic-boundary-conditions. Two of them should be particularly made aware of, because they are artifacts of the modeling and can be avoided or at least minimized by proper modeling. The first is the depolarization (deionization) that can emerge due to use of small supercells in DFT calculations and consequently unreasonably high concentration of ions. The second is due to periodic-boundary-conditions applied along lateral directions and, for multi-slab models, also along the surface normal direction.

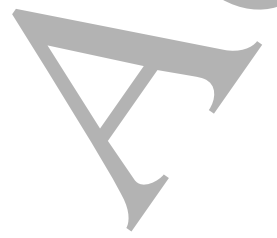




\section{Acknowledgments}

Authors acknowledge helpful discussions with Fatah Chiter and Matic Poberžnik. This work is a part of M-ERA.NET project entitled "Design of corrosion resistant coatings targeted for versatile applications" (acronym COR_ID). The financial support of the project by MESS (Ministry of Education, Science and Sport of Republic of Slovenia), Grant No. 3330-16-500040, and ANR (The French National Research Agency), Grant No. ANR-15-MERA-0004, is acknowledged. This work has been also supported by the Slovenian Research Agency (Grant No. P2-0393).

\section{Appendix A. A single point-charge near an interface between two dielectrics}

Here we present some basic equations on which equations, used in the Theory section, are based. Let us consider a model system composed of two semi-infinite dielectrics, characterized by $\varepsilon_{1}$ and $\varepsilon_{2}$, and a point-charge $q$ located in dielectric- 1 near the planar interface between the two dielectrics (beware that in other sections $q$ instead designates the magnitude of the pointcharge). Let the charge be located at $\left(R, z_{0}\right)$ in cylindrical coordinates with $z_{0}>0$ and let the interface lie on the $z=0$ plane (Fig. A.1). In this case the electric potential can be obtained by the method of images and the derivation closely follows Ref. 38. The electric potential in dielectric- 1 at point $(0, z>0)$ can be written as:

$$
\phi(0, z>0)=\frac{1}{\varepsilon_{1}}\left(\frac{q}{R_{z_{0}-z}}+\frac{q^{\prime}}{R_{z_{0}+z}}\right)
$$

where

$$
R_{z_{0}-z}=\sqrt{R^{2}+\left(z_{0}-z\right)^{2}} \text { and } R_{z_{0}+z}=\sqrt{R^{2}+\left(z_{0}+z\right)^{2}}
$$

where $q^{\prime}$ stands for the image-charge of a magnitude that will be determined by solving the problem. The electric potential in dielectric- 2 can be written as:

$$
\phi(0, z<0)=\frac{1}{\varepsilon_{2}}\left(\frac{q^{\prime \prime}}{R_{z_{0}-z}}\right),
$$

where the magnitude of $q^{\prime \prime}$ is to be determined (notice that there is no image-charge counterpart in this case, because there are no charges ${ }^{\|}$in dielectric-2). The $q^{\prime}$ and $q^{\prime \prime}$ can be obtained by considering the boundary conditions. At the interface there are no free charges and therefore no discontinuities, hence approaching the interface from above $\left(z \rightarrow 0_{+}\right)$or from below $\left(z \rightarrow 0_{-}\right)$ should yield the same results, i.e.:

$$
\phi\left(0_{+}\right)=\phi\left(0_{-}\right) \quad \text { and } \quad \varepsilon_{1} \mathcal{E}_{z}\left(0_{+}\right)=\varepsilon_{2} \mathcal{E}_{z}\left(0_{-}\right)
$$

where $\phi$ is the electric potential and $\mathcal{E}_{z}$ is the $z$-component of the electric field (note that $\mathcal{E}_{z}=$ $-\partial \phi / \partial z$ ). Solving Eqs. [A.1] and [A.3] subjected to boundary conditions of Eq. [A.4] gives: ${ }^{38}$

$$
q^{\prime}=\frac{\varepsilon_{1}-\varepsilon_{2}}{\varepsilon_{1}+\varepsilon_{2}} q \quad \text { and } \quad q^{\prime \prime}=\frac{2 \varepsilon_{2}}{\varepsilon_{1}+\varepsilon_{2}} q
$$

${ }^{\|}$Here only the so-called free charges are meant without the polarization charge that develops at the interface. 

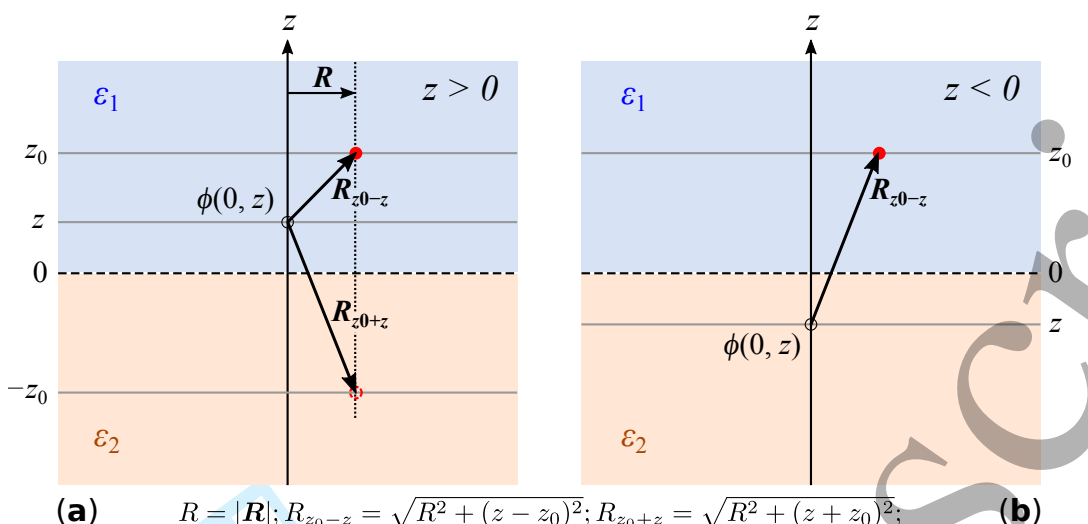

(a)

$R=|\boldsymbol{R}| ; R_{z_{0}-z}=\sqrt{R^{2}+\left(z-z_{0}\right)^{2}} ; R_{z_{0}+z}=\sqrt{R^{2}+\left(z+z_{0}\right)^{2}} ;$

Figure A.1. Electric potential at point $(0, z)$ for a point-charge located in the dielectric-1 near the interface between the two dielectrics, characterized by $\varepsilon_{1}$ and $\varepsilon_{2}$, can be obtained by the method of images. Interface is located at $z=0$, dielectric-1 at $z>0$, and dielectric-2 at $z<0$. (a) Scheme for the potential at $z>0$ in dielectric-1 and (b) the corresponding scheme for the potential at $z<0$ in dielectric- 2 .

where the following relations were used:

$$
\mathcal{E}_{z}=-\nabla \phi=-\frac{\partial \phi}{\partial z} \quad \text { and } \quad \frac{\partial R_{z_{0}-z}^{-1}}{\partial z}=\frac{\partial\left(R^{2}+\left(z_{0}-z\right)^{2}\right)^{-\frac{1}{2}}}{\partial z}=\frac{z_{0}-z}{R_{z_{0}-z}^{3}}
$$

By utilizing Eqs. [A.1], [A.3], and [A.5], the electric potential in dielectric-1 at point $(0, z>0)$ is therefore given by:

$$
\phi(0, z>0)=\frac{q}{\varepsilon_{1}}\left(\frac{1}{R_{z_{0}-z}}+\frac{\varepsilon_{1}-\varepsilon_{2}}{\varepsilon_{1}+\varepsilon_{2}} \frac{1}{R_{z_{0}+z}}\right)
$$

and the potential in dielectric- 2 at $(0, z<0)$ is given by:

$$
\phi(0, z<0)=\frac{2 q}{\varepsilon_{1}+\varepsilon_{2}}\left(\frac{1}{R_{z_{0}-z}}\right) .
$$

It is worth noting that Eqs. [7], [8], [11], and [12] in the Theory section are based on Eqs. [A.7] and [A.8].

\section{Appendix B. Slope of the electrostatic interaction energy with respect to the interlayer distance}

Electrostatic interaction energy for two infinite parallel layers of point charges near the interface between two dielectrics are given by Eqs. [10] and [13] that are defined in terms of electric potentials $\phi_{11}$ and $\phi_{12}$, i.e.:

$$
\begin{aligned}
U_{11}(z) & =\frac{1}{2}\left(q^{+} \phi_{11}\left(0,0, z_{0}\right)+q^{-} \phi_{11}(0,0, z)\right) \\
& =\frac{q^{2}}{2 \varepsilon_{1}}\left\{\sum_{R \neq 0}\left[\frac{2}{R}+\frac{\varepsilon_{1}-\varepsilon_{2}}{\varepsilon_{1}+\varepsilon_{2}}\left(\frac{1}{R_{2 z_{0}}}+\frac{1}{R_{2 z}}\right)\right]-\sum_{R}\left[\frac{2}{R_{z_{0}-z}}+\frac{\varepsilon_{1}-\varepsilon_{2}}{\varepsilon_{1}+\varepsilon_{2}}\left(\frac{2}{R_{z_{0}+z}}\right)\right]\right\}
\end{aligned}
$$


and

$$
\begin{aligned}
U_{12}(z)= & \frac{1}{2}\left(q^{+} \phi_{12}\left(0,0, z_{0}\right)+q^{-} \phi_{12}(0,0, z)\right) \\
=\frac{q^{2}}{2} & \left\{\sum_{\boldsymbol{R} \neq 0} \frac{1}{\varepsilon_{1}}\left(\frac{1}{R}+\frac{\varepsilon_{1}-\varepsilon_{2}}{\varepsilon_{1}+\varepsilon_{2}} \frac{1}{R_{2 z_{0}}}\right)-\frac{2}{\varepsilon_{1}+\varepsilon_{2}} \sum_{\boldsymbol{R}} \frac{1}{R_{z_{0}-z}}\right. \\
& \left.+\sum_{\boldsymbol{R} \neq 0} \frac{1}{\varepsilon_{2}}\left(\frac{1}{R}+\frac{\varepsilon_{2}-\varepsilon_{1}}{\varepsilon_{1}+\varepsilon_{2}} \frac{1}{R_{-2 z}}\right)-\frac{2}{\varepsilon_{1}+\varepsilon_{2}} \sum_{\boldsymbol{R}} \frac{1}{R_{z_{0}-z}}\right\},
\end{aligned}
$$

where $q$ is the magnitude of a point charge, $q=q^{+}=-q^{-}$. These equations involve infinite lattice sums and therefore their dependence on the interlayer distance is not trivially obvious. If one is only interested in the slope of the interaction energy with respect to the interlayer distance then these two equations can be simplified by approximating the lattice sums by integrals. For example:

$$
\sum_{\boldsymbol{R}=\mathbf{0}}^{\boldsymbol{R}_{\max }} \frac{q}{R_{z_{0}-z}} \approx 2 \pi \sigma \int_{0}^{R_{\max }} \frac{R}{R_{z_{0}-z}} \mathrm{~d} R=2 \pi \sigma\left(R_{z_{0}-z}^{\max }-\left(z_{0}-z\right)\right) .
$$

where $\sigma$ is the magnitude of the surface charge density, $\sigma=q / A$, and $A$ is the area of the $2 \mathrm{D}$ unitcell (or supercell). The following limits will be used below:

$$
\begin{aligned}
& \lim _{R \rightarrow 0} R_{z_{0}-z}=\lim _{R \rightarrow 0} \sqrt{R^{2}+\left(z_{0}-z\right)^{2}}=z_{0}-z, \\
& \lim _{R \rightarrow \infty} R_{z_{0}-z}=\lim _{R \rightarrow \infty} \sqrt{R^{2}+\left(z_{0}-z\right)^{2}}=R .
\end{aligned}
$$

But beware that:

$$
\lim _{R \rightarrow 0} R_{-2 z}=\lim _{R \rightarrow 0} \sqrt{R^{2}+(-2 z)^{2}}=-2 z,
$$

because $R_{-2 z}$ is used when $z<0$ (see Fig. 5) and, thus, $-2 z>0$ (note that the subscript of $R$ stands for the distance between the two layers and a distance is never negative).

Using the integrals to approximate the sums, the interaction energy $U_{11}$ of Eq. [B.1] can be approximated as:

$$
U_{11}^{\text {approx }}=\frac{\pi q^{2}}{\varepsilon_{1} A} \int_{0}^{\infty}\left[2+\frac{\varepsilon_{1}-\varepsilon_{2}}{\varepsilon_{1}+\varepsilon_{2}}\left(\frac{R}{R_{2 z_{0}}}+\frac{R}{R_{2 z}}\right)-\frac{2 R}{R_{z_{0}-z}}-\frac{\varepsilon_{1}-\varepsilon_{2}}{\varepsilon_{1}+\varepsilon_{2}}\left(\frac{2 R}{R_{z_{0}+z}}\right)\right] \mathrm{d} R .
$$

By using Eqs. [B.3]-[B.5], this integral is equal to:

$$
\begin{array}{r}
U_{11}^{\text {approx }}=\frac{\pi q^{2}}{\varepsilon_{1} A}\left\{\lim _{R \rightarrow \infty}\left[2 R+2 \frac{\varepsilon_{1}-\varepsilon_{2}}{\varepsilon_{1}+\varepsilon_{2}} R-2 R-2 \frac{\varepsilon_{1}-\varepsilon_{2}}{\varepsilon_{1}+\varepsilon_{2}} R\right]\right. \\
\left.-\left[\frac{\varepsilon_{1}-\varepsilon_{2}}{\varepsilon_{1}+\varepsilon_{2}}\left(2 z_{0}+2 z\right)-2\left(z_{0}-z\right)-2 \frac{\varepsilon_{1}-\varepsilon_{2}}{\varepsilon_{1}+\varepsilon_{2}}\left(z_{0}+z\right)\right]\right\} .
\end{array}
$$

The part in the first square-brackets is 0 , whereas the part in the second square-brackets is:

$$
2 \frac{\left(\varepsilon_{1}-\varepsilon_{2}\right)\left(z_{0}+z\right)-\left(\varepsilon_{1}+\varepsilon_{2}\right)\left(z_{0}-z\right)-\left(\varepsilon_{1}-\varepsilon_{2}\right)\left(z_{0}+z\right)}{\varepsilon_{1}+\varepsilon_{2}}=-2\left(z_{0}-z\right) .
$$

Hence:

$$
U_{11}^{\text {approx }}=\frac{2 \pi q^{2}}{A \varepsilon_{1}}\left(z_{0}-z\right) \quad \text { and } \quad \frac{\partial U_{11}^{\text {approx }}}{\partial z}=-\frac{2 \pi q^{2}}{A \varepsilon_{1}} \propto-\frac{\sigma}{\varepsilon_{1}},
$$


which is the result used by Eqs. [16] and [17] in the Theory section. Note that according to this equation $U_{11}^{\text {approx }}=0$ for $z=z_{0}$. This is because $U_{11}^{\text {approx }}$ measures only the interaction between the two plates (per area $A$ ) and at $z=z_{0}$ the two plates merge and the charges annihilate.

Analogously, $U_{12}$ of Eq. [B.2] can be approximated as:

$$
\begin{aligned}
U_{12}^{\text {approx }}=\frac{\pi q^{2}}{A} \int_{0}^{\infty}( & \left.\frac{1}{\varepsilon_{1}}\left[1+\frac{\varepsilon_{1}-\varepsilon_{2}}{\varepsilon_{1}+\varepsilon_{2}} \frac{R}{R_{2 z_{0}}}\right]-\frac{2}{\varepsilon_{1}+\varepsilon_{2}} \frac{R}{R_{z_{0}-z}}\right)+ \\
& \left(+\frac{1}{\varepsilon_{2}}\left[1+\frac{\varepsilon_{2}-\varepsilon_{1}}{\varepsilon_{1}+\varepsilon_{2}} \frac{R}{R_{-2 z}}\right]-\frac{2}{\varepsilon_{1}+\varepsilon_{2}} \frac{R}{R_{z_{0}-z}}\right) \mathrm{d} R .
\end{aligned}
$$

After the integration the $R \rightarrow \infty$ part of the integral is:

$$
\left(\frac{1}{\varepsilon_{1}}\left[1+\frac{\varepsilon_{1}-\varepsilon_{2}}{\varepsilon_{1}+\varepsilon_{2}}\right] R-\frac{2}{\varepsilon_{1}+\varepsilon_{2}} R\right)+\left(\frac{1}{\varepsilon_{2}}\left[1+\frac{\varepsilon_{2}-\varepsilon_{1}}{\varepsilon_{1}+\varepsilon_{2}}\right] R-\frac{2}{\varepsilon_{1}+\varepsilon_{2}} R\right)=0,
$$

which can be straightforwardly foreseen by noticing that:

$$
1+\frac{\varepsilon_{1}-\varepsilon_{2}}{\varepsilon_{1}+\varepsilon_{2}}=\frac{2 \varepsilon_{1}}{\varepsilon_{1}+\varepsilon_{2}} \quad \text { and } \quad 1+\frac{\varepsilon_{2}-\varepsilon_{1}}{\varepsilon_{1}+\varepsilon_{2}}=\frac{2 \varepsilon_{2}}{\varepsilon_{1}+\varepsilon_{2}} .
$$

Only the $R=0$ part therefore remains after the integration. Hence:

$$
U_{12}^{\text {approx }}=-\frac{2 \pi q^{2}}{A}\left(\frac{1}{\varepsilon_{1}} \frac{\varepsilon_{1}-\varepsilon_{2}}{\varepsilon_{1}+\varepsilon_{2}} z_{0}-\frac{1}{\varepsilon_{1}+\varepsilon_{2}}\left(z_{0}-z\right)+\frac{1}{\varepsilon_{2}} \frac{\varepsilon_{2}-\varepsilon_{1}}{\varepsilon_{1}+\varepsilon_{2}}(-z)-\frac{1}{\varepsilon_{1}+\varepsilon_{2}}\left(z_{0}-z\right)\right) .
$$

The part in the brackets is:

$$
\left(\frac{1}{\varepsilon_{1}} \frac{\varepsilon_{1}-\varepsilon_{2}}{\varepsilon_{1}+\varepsilon_{2}}-\frac{2}{\varepsilon_{1}+\varepsilon_{2}}\right) z_{0}+\left(\frac{1}{\varepsilon_{2}} \frac{\varepsilon_{1}-\varepsilon_{2}}{\varepsilon_{1}+\varepsilon_{2}}+\frac{2}{\varepsilon_{1}+\varepsilon_{2}}\right) z=-\frac{1}{\varepsilon_{1}} z_{0}+\frac{1}{\varepsilon_{2}} z
$$

and therefore:

$$
U_{12}^{\text {approx }}=\frac{2 \pi q^{2}}{A}\left(\frac{z_{0}}{\varepsilon_{1}}-\frac{z}{\varepsilon_{2}}\right) y \quad \text { and } \quad \frac{\partial U_{12}^{\text {approx }}}{\partial z}=-\frac{2 \pi q^{2}}{A \varepsilon_{2}} \propto-\frac{\sigma}{\varepsilon_{2}},
$$

which is the result used by Eqs. [16] and [17] in the Theory section.

It should be noted that the negative slopes of $\partial U_{11}^{\text {approx }} / \partial z$ and $\partial U_{12}^{\text {approx }} / \partial z$ are the consequence of the setup, i.e., the layer of positive charges fixed at $z_{0}$ and the layer of negative charges at $z<z_{0}$ moving away from it in the $-z$ direction. The negative slope is therefore the consequence of the "movement" in the negative direction. The main message is thus that the larger is the interlayer distance, the more positive (endothermic) is the energy.

\section{Appendix C. Dependence of the activation barrier for penetration of $\mathrm{Na}^{+} / \mathrm{Cl}^{-}$ion-pair into SAM on the electric field}

The electric field $\mathcal{E}$ can stabilize the ion-pair (described by a dipole $\boldsymbol{\mu}$ ) by $-\mathcal{E} \cdot \boldsymbol{\mu}$ and the approximate Eq. [40] in the main text suggests that for strong enough electric fields (or surface charge densities), the barrier for the penetration of $\mathrm{Na}^{+} / \mathrm{Cl}^{-}$ion-pair into SAM would drop to zero. However, the electric field takes the full effect only when the ion-pair is fully in SAM, 

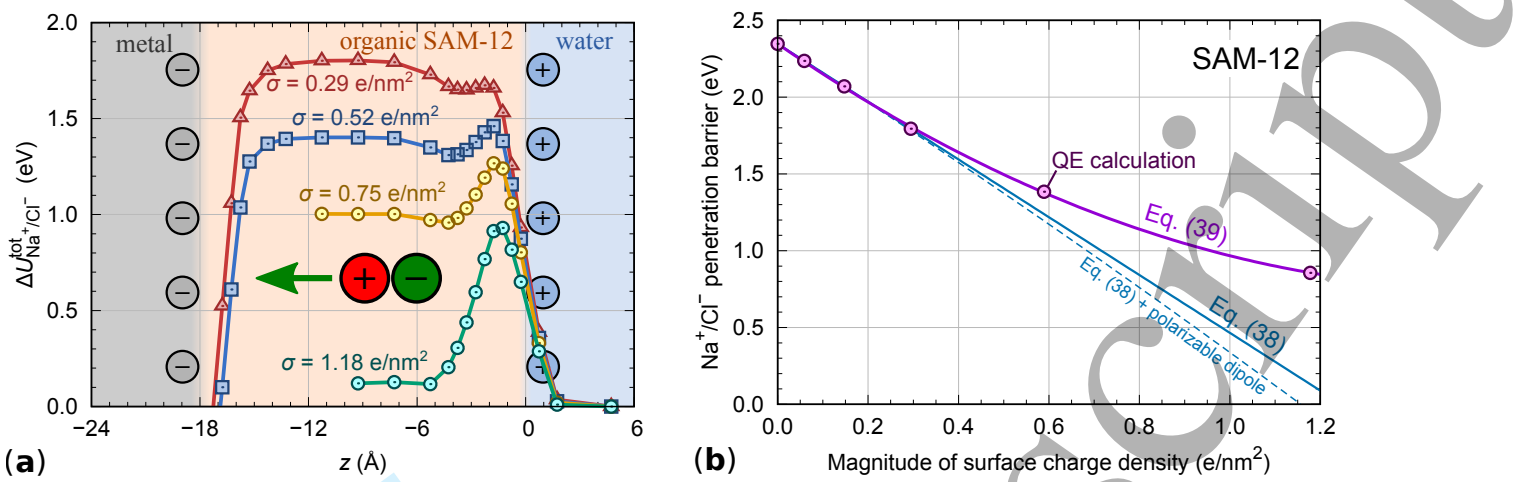

Figure C.2. (a) QE calculated energy profiles for the penetration of the $\mathrm{Na}^{+} / \mathrm{Cl}^{-}$ion-pair from water though SAM-12 toward the metal surface for several metal surface charge densities $\sigma$. Note the emergence of "extra solvation energy penalty" as the magnitude of metal surface charge density increases. (b) QE calculated activation energies (data points) for the penetration of $\mathrm{Na}^{+} / \mathrm{Cl}^{-}$ion-pair though SAM-12 as a function of metal surface charge density. The fit of QE data points with Eq. [41] is indicated by purple line, whereas bluish line indicates the prediction of Eq. [40]; the case of polarizable $\mathrm{Na}^{+} / \mathrm{Cl}^{-}$dipole, Eq. [C.3], is indicated by dashed curve, where the PBE calculated value of $\alpha=30 \mathrm{Bohr}^{3}$ was taken for the polarizability of the $\mathrm{Na}^{+} / \mathrm{Cl}^{-}$ion-pair.

but the change in solvation from water to SAM kicks in prior to that and, correspondingly, the penetration barrier remains non-vanishing even for electric fields that are stronger than those for which Eq. [40] predicts no barrier. This phenomenon is shown in Fig. C.2a, which displays the energy profiles for the penetration of the $\mathrm{Na}^{+} / \mathrm{Cl}^{-}$ion-pair from water through SAM-12 toward the metal surface for several metal surface charge densities. The aforementioned emergence of extra "solvation energy penalty" as the magnitude of metal surface charge density increases is clearly evident. For this reason the penetration barriers at large surface charge density magnitudes are larger than those predicted by Eq. [40] and this is evident from Fig C.2b, which compares QE calculated energy barriers to those predicted by Eq. [40]. Note that the QE data points are very well fitted with the quadratic ansatz of Eq. [41]. Finally let us mention that if the $\mathrm{Na}^{+} / \mathrm{Cl}^{-}$dipole is considered as polarizable then the dependence on the electric field is also (slightly) quadratic but with the opposite effect to that of Eq [41], that is, polarizability further stabilizes the dipole in the electric field and in turn reduces the barrier with respect to Eq. [40]. In particular, the relation between electric field $\mathcal{E}$ and polarizable dipole $\boldsymbol{\mu}$ can be written as:

$$
\boldsymbol{\mu}=\boldsymbol{\mu}_{0}+\alpha \mathcal{E},
$$

where $\boldsymbol{\mu}_{0}$ is the zero-field dipole and $\alpha$ is the dipole polarizability. Note that for polarizable dipole, the electric field stabilization is not given by $-\boldsymbol{\mu} \cdot \mathcal{E}$, because some energy is expended to polarize the dipole and the corresponding polarization energy ( $\left.U_{\text {polar }}\right)$ is given by: ${ }^{39,40}$

$$
U_{\text {polar }}=\frac{\left(\boldsymbol{\mu}-\boldsymbol{\mu}_{0}\right)^{2}}{2 \alpha}=\frac{1}{2} \alpha \mathcal{E}^{2} .
$$

With aid of Eqs. [C.1] and [C.2], the stabilization of polarizable dipole by the electric field can be written as:

$$
U_{\text {dipole }}=-\boldsymbol{\mu} \cdot \mathcal{E}+\frac{1}{2} \alpha \mathcal{E}^{2}=-\boldsymbol{\mu}_{0} \cdot \mathcal{E}-\frac{1}{2} \alpha \mathcal{E}^{2},
$$

which implies that the electric field induced polarization of the dipole stabilizes it by $\frac{1}{2} \alpha \mathcal{E}^{2}$ with respect to the non-polarizable dipole $\mu_{0}$ (see Fig. C.2b). 


\section{References}

1. G. S. Frankel, Pitting corrosion of metals: A review of the critical factors, J. Electrochem. Soc. 145, 2186-2198 (1998).

2. D. Costa, T. Ribeiro, P. Cornette, and P. Marcus, DFT modeling of corrosion inhibition by organic molecules: Carboxylates as inhibitors of aluminum corrosion, J. Phys. Chem. C 120, 28607-28616 (2016).

3. M. Poberžnik, D. Costa, A. Hemeryck, and A. Kokalj, Insight into the bonding of silanols to oxidized aluminum surfaces, J. Phys. Chem. C 122, 9417-9431 (2018).

4. M. Poberžnik and A. Kokalj, Implausibility of bidentate bonding of the silanol headgroup to oxidized aluminum surfaces, Appl. Surf. Sci. 492, 909-918 (2019).

5. I. Milošev, D. Zimerl, C. Carriére, S. Zanna, A. Seyeux, J. Iskra, S. Stavber, F. Chiter, M. Poberžnik, D. Costa, A. Kokalj, and P. Marcus, Editors' choice-The effect of anchor group and alkyl backbone chain on performance of organic compounds as corrosion inhibitors for aluminum investigated using an integrative experimental-modeling approach, J. Electrochem. Soc. 167, 061509 (2020).

6. I. Milošev, T. Bakarič, S. Zanna, A. Seyeux, P. Rodič, M. Poberžnik, F. Chiter, P. Cornette, D. Costa, A. Kokalj, and P. Marcus, Electrochemical, surface-analytical, and computational DFT study of alkaline etched aluminum modified by carboxylic acids for corrosion protection and hydrophobicity, J. Electrochem, Soc. 166, C3131 (2019).

7. M. Poberžnik, F. Chiter, I. Milošev, P. Marcus, D. Costa, and A. Kokalj, DFT study of $n$-alkyl carboxylic acids on oxidized aluminum surfaces: from standalone molecules to self-assembled-monolayers, Appl. Surf. Sci. 525, 146156 (2020).

8. I. Milošev, A. Kokalj, M. Poberžnik, C. Carrière, D. Zimerl, J. Iskra, A. Nemes, D. Szabó, S. Zanna, A. Seyeux, D. Costa, J. Rábai, and P. Marcus, The effects of perfluoroalkyl and alkyl backbone chains, spacers, and anchor groups on the performance of organic compounds as corrosion inhibitors for aluminum investigated using an integrative experimental-modeling approach, J. Electrochem. Soc. (2021), submitted.

9. P. Marcus, V. Maurice, and H.-H. Strehblow, Localized corrosion (pitting): A model of passivity breakdown including the role of the oxide layer nanostructure, Corros. Sci. 50, 2698-2704 (2008).

10. P. M. Natishan and W. E. O'Grady, Chloride ion interactions with oxide-covered aluminum leading to pitting corrosion: A review, J. Electrochem. Soc. 161, C421-C432 (2014).

11. J. Soltis, Passivity breakdown, pit initiation and propagation of pits in metallic materials - review, Corros. Sci. 90, 5-22 (2015).

12. E. McCafferty, Sequence of steps in the pitting of aluminum by chloride ions, Corros. Sci. 45, 1421-1438 (2003).

13. K. Leung, First principles, explicit interface studies of oxygen vacancy and chloride in alumina films for corrosion applications, J. Electrochem. Soc. 168, 031511 (2021).

14. H. Ke, G. S. Frankel, and C. D. Taylor, Application of the chloride susceptibility index to study the effects of Ni, $\mathrm{Cr}, \mathrm{Mn}$ and Mo on the repassivation of stainless steels, J. Electrochem. Soc. 167, 131510 (2020).

15. L. Kleinman, Comment on the average potential of a wigner solid, Phys. Rev. B 24, 7412-7414 (1981).

16. P. Giannozzi, O. Andreussi, T. Brumme, O. Bunau, M. B. Nardelli, M. Calandra, R. Car, C. Cavazzoni, D. Ceresoli, M. Cococcioni, N. Colonna, I. Carnimeo, A. D. Corso, S. de Gironcoli, P. Delugas, R. DiStasio, A. Ferretti, A. Floris, G. Fratesi, G. Fugallo, R. Gebauer, U. Gerstmann, F. Giustino, T. Gorni, J. Jia, M. Kawamura, H.-Y. Ko, A. Kokalj, E. Küçükbenli, M. Lazzeri, M. Marsili, N. Marzari, F. Mauri, N. L. Nguyen, H.-V. Nguyen, A. O. de-la Roza, L. Paulatto, S. Poncé, D. Rocca, R. Sabatini, B. Santra, M. Schlipf, A. P. Seitsonen, A. Smogunov, I. Timrov, T. Thonhauser, P. Umari, N. Vast, X. Wu, and S. Baroni, Advanced capabilities for materials modelling with quantum espresso, J. Phys: Condens. Matter 29, 465901 (2017).

17. P. E. Blöchl, Projector augmented-wave method, Phys. Rev. B 50, 17953-17979 (1994).

18. We used PAW potentials from the pslibrary, ${ }^{41}$ in particular: Na.pbe-spn-kjpaw_ps1.0.2.UPF, Cl.pbe-n-kjpaw_psl.1.0.0.UPF, and He.pbe-kjpaw_psl.1.0.0.UPF.

19. J. P. Perdew, K. Burke, and M. Ernzerhof, Generalized gradient approximation made simple, Phys. Rev. Lett. 77, 3865-3868 (1996).

20. F. Chiter, M. Poberžnik, A. Kokalj, I. Milošev, P. Marcus, and D. Costa, Cl ingress into carboxylate layers on al studied by dft: A new descriptor of corrosion inhibition by organic inhibitors, to be submitted (2021).

21. S. Grimme, Semiempirical GGA-type density functional constructed with a long-range dispersion correction, $J$. Comput. Chem. 27, 1787-1799 (2006).

22. O. Andreussi, I. Dabo, and N. Marzari, Revised self-consistent continuum solvation in electronic-structure calculations, J. Chem. Phys. 136,064102 (2012).

23. M. J. Frisch, G. W. Trucks, H. B. Schlegel, G. E. Scuseria, M. A. Robb, J. R. Cheeseman, G. Scalmani, V. Barone, G. A. Petersson, H. Nakatsuji, X. Li, M. Caricato, A. V. Marenich, J. Bloino, B. G. Janesko, R. Gomperts, B. Mennucci, H. P. Hratchian, J. V. Ortiz, A. F. Izmaylov, J. L. Sonnenberg, D. Williams-Young, F. Ding, F. Lipparini, F. Egidi, J. Goings, B. Peng, A. Petrone, T. Henderson, D. Ranasinghe, V. G. Zakrzewski, J. Gao, N. Rega, G. Zheng, W. Liang, M. Hada, M. Ehara, K. Toyota, R. Fukuda, J. Hasegawa, M. Ishida, T. Nakajima, Y. Honda, O. Kitao,

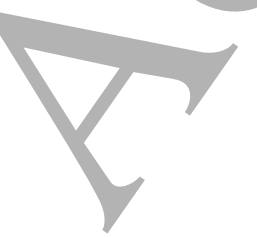


H. Nakai, T. Vreven, K. Throssell, J. A. Montgomery, Jr., J. E. Peralta, F. Ogliaro, M. J. Bearpark, J. J. Heyd, E. N. Brothers, K. N. Kudin, V. N. Staroverov, T. A. Keith, R. Kobayashi, J. Normand, K. Raghavachari, A. P. Rendell, J. C. Burant, S. S. Iyengar, J. Tomasi, M. Cossi, J. M. Millam, M. Klene, C. Adamo, R. Cammi, J. W. Ochterski, R. L. Martin, K. Morokuma, O. Farkas, J. B. Foresman, and D. J. Fox, Gaussian 16 Revision A.03, 2016, Gaussian Inc. Wallingford CT.

24. A. V. Marenich, C. J. Cramer, and D. G. Truhlar, Universal solvation model based on solute electron density and on a continuum model of the solvent defined by the bulk dielectric constant and atomic surface tensions, J. Phys. Chem. B 113, 6378-6396 (2009).

25. F. Weigend and R. Ahlrichs, Balanced basis sets of split valence, triple zeta valence and quadruple zeta valence quality for h to rn: Design and assessment of accuracy, Phys. Chem. Chem. Phys. 7, 3297-3305 (2005).

26. S. Baroni, S. de Gironcoli, A. Dal Corso, and P. Giannozzi, Phonons and related crystal properties from densityfunctional perturbation theory, Rev. Mod. Phys. 73, 515-562 (2001).

27. T. Williams, C. Kelley, and many others, Gnuplot 5.2, 2019, http://www.gnuplot.info/.

28. Inkscape Project, Inkscape, 2019, version 0.92.4.

29. A. Kokalj, XCrySDen-a new program for displaying crystalline structures and electron densities, J. Mol. Graph. Model. 17, 176-179 (1999), Code available from http://www.xcrysden.org/.

30. G. Fisicaro, L. Genovese, O. Andreussi, S. Mandal, N. N. Nair, N. Marzari, and S. Goedecker, Soft-Sphere Continuum Solvation in Electronic-Structure Calculations, J. Chem. Theory Comput. 13, 3829-3845 (2017).

31. W. Schmickler and E. Santos, Interfacial electrochemistry, Springer Science \& Business Media, Verlag Berlin Heidelberg, 2nd edition, 2010.

32. D. R. Lide, editor, CRC Handbook of Chemistry and Physics, CRC Press, Boca Raton, Florida USA, 85th edition, 2005 .

33. M. Poberžnik and A. Kokalj, Surprising lateral interactions between negatively charged adatoms on metal surfaces, J. Phys. Chem. Lett. 11, 7122-7126 (2020).

34. W. P. Yang, D. Costa, and P. Marcus, Resistance to pitting and chemical composition of passive films of a Fe- $17 \% \mathrm{Cr}$ alloy in chloride-containing acid solution, J. Electrochem. Soc. 141, 2669 (1994).

35. C.-O. A. Olsson, P. Agarwal, M. Frey, and D. Landolt, An XPS study of the adsorption of organic inhibitors on mild steel surfaces, Corros. Sci. 42, 1197-1211 (2000).

36. T. Hashemi and C. A. Hogarth, The mechanism of corrosion inhibition of copper in nacl solution by benzotriazole studied by electron spectroscopy, Electrochim. Acta 33, 1123-1127 (1988).

37. D. K. Kozlica, A. Kokalj, and I. Milošev, Synergistic effect of 2-mercaptobenzimidazole and octylphosphonic acid as corrosion inhibitors for copper and aluminium - An electrochemical, XPS, FTIR and DFT study, Corros. Sci. 182, 109082 (2021).

38. R. Fitzpatrick, Classical electromagnetism, The University of Texas at Austin, Austin (2006), Last accessed on Jan 2021 at http://farside.ph.utexas.edu/teaching/jk1/jk1.html.

39. B. L. Maschhoff and J. P. Cowin, Corrected electrostatic model for dipoles adsorbed on a metal surface, J. Chem. Phys. 101, 8138-8151 (1994).

40. A. Kokalj, Electrostatic model for treating long-range lateral interactions between polar molecules adsorbed on metal surfaces, Phys. Rev. B 84, 045418 (2011).

41. A. Dal Corso, Pseudopotentials periodic table: From H to Pu, Comp. Mater. Sci. 95, 337-350 (2014). 